\title{
Vector valued reproducing kernel Hilbert spaces and universality
}

\author{
C. Carmeli, , E. De Vitoł A. Toigo
}

October 22, 2018

\begin{abstract}
This paper is devoted to the study of vector valued reproducing kernel Hilbert spaces. We focus on two aspects: vector valued feature maps and universal kernels. In particular we characterize the structure of translation invariant kernels on abelian groups and we relate it to the universality problem.
\end{abstract}

\section{Introduction}

In learning theory, reproducing kernel Hilbert spaces (RKHS) are an important tool for designing learning algorithms, see for example [8, 29, 31] and the book [9]. In the usual setting the elements of the RKHS are scalar functions. The mathematical theory for scalar RKHS has been established in the seminal paper [1]. For a standard reference see the book [25].

In machine learning there is an increasing interest for vector valued learning algorithms, see [20, 12, 4]. In this framework, the basic object is a Hilbert space of functions $f$ from a set $X$ into a normed vector space $\mathcal{Y}$ with the property that, for any $x \in X,\|f(x)\| \leq C_{x}\|f\|$ for a positive constant $C_{x}$

${ }^{*}$ C. Carmeli, DIFI, Università di Genova, and I.N.F.N., Sezione di Genova, Via Dodecaneso 33, 16146 Genova, Italy. e-mail: carmeli@ge.infn.it

${ }^{\dagger}$ E. De Vito, DSA., Università di Genova, Stradone S. Agostino 37, 16123 Genova, Italy, and I.N.F.N., Sezione di Genova, Via Dodecaneso 33, 16146 Genova, Italy. e-mail: devito@dima.unige.it

${ }^{\ddagger}$ A. Toigo, DISI, Università di Genova, Via Dodecaneso 35, 16146 Genova, and I.N.F.N., Sezione di Genova, Via Dodecaneso 33, 16146 Genova, Italy. e-mail: toigo@ge.infn.it

$\S$ V. Umanità, DISI, Università di Genova, Via Dodecaneso 35, 16146 Genova, and Dipartimento di Matematica "F. Brioschi", Politecnico di Milano, Piazza Leonardo da Vinci 32, I-20133 Milano, Italy. e-mail: veronica.umanita@polimi.it 
independent of $f$.

The theory of vector valued RKHS has been completely worked out in the seminal paper [27], devoted to the characterization of the Hilbert spaces that are continuously embedded into a locally convex topological vector space, see [23]. In the case $\mathcal{Y}$ is itself a Hilbert space, the theory can be simplified as shown in [21, 6, 5]. As in the scalar case, a RKHS is completely characterize by a map $K$ from $X \times X$ into the space of bounded operators on $\mathcal{Y}$ such that

$$
\sum_{i, j=1}^{N}\left\langle K\left(x_{i}, x_{j}\right) y_{j}, y_{i}\right\rangle \geq 0
$$

for any $x_{1}, \ldots, x_{N}$ in $X$ and $y_{1}, \ldots, y_{N}$ in $\mathcal{Y}$. Such a map is called a $\mathcal{Y}$ reproducing kernel and the corresponding RKHS is denoted by $\mathcal{H}_{K}$.

This paper focuses on three aspects of particular interest in vector valued learning problems:

- vector valued feature maps;

- universal reproducing kernels;

- translation invariant reproducing kernels.

The feature map approach is the standard way in which scalar RKHS are presented in learning theory, see for example [26]. A feature map is a function mapping the input space $X$ into an arbitrary Hilbert space $\mathcal{H}$ in such a way that $\mathcal{H}$ can be identified with a unique RKHS. Conversely, any RKHS can be realized as a closed subspace of a concrete Hilbert space, called feature space, by means of a suitable feature map - typical examples of feature spaces are $\ell^{2}$ and $L^{2}(X, \mu)$ for some measure $\mu$.

The concept of feature map is extended to the vector valued setting in [6, 5], where a feature map is defined as a function from $X$ into the space of bounded operators between $\mathcal{Y}$ and the feature space $\mathcal{H}$.

In the first part of our paper, Section 3 shows that sum, product and composition with maps of RKHS can be easily described by suitable feature maps. In particular we give an elementary proof of Schur lemma about the product of a scalar kernel with a vector valued kernel. Moreover, we present several examples of vector valued RKHS, most of them considered in [22, 5]. For each one of them we exhibit a nice feature space. This allows to describe the impact of these examples on some learning algorithms, like the regularized least-squares [13].

In the second part of the paper, Section 4 discusses the problem of characterizing universal kernels. We say that a $\mathcal{Y}$-reproducing kernel is universal 
if the corresponding RKHS $\mathcal{H}_{K}$ is dense in $L^{2}(X, \mu ; \mathcal{Y})$ for any probability measure $\mu$ on the input space $X$. This definition is motivated observing that in learning theory the goal is to approximate a target function $f^{*}$ by means of a prediction function $f_{n} \in \mathcal{H}_{K}$, depending on the data, in such a way the distance between $f^{*}$ and $f_{n}$ goes to zero when the number of data $n$ goes to infinity. In learning theory the "right" distance is given by the norm in $L^{2}(X, \mu ; \mathcal{Y})$, where $\mu$ is the (unknown) probability distribution modeling the sample of the input data, see [8]. The possibility of learning any target function $f^{*}$ by means of functions in $\mathcal{H}_{K}$ is precisely the density of $\mathcal{H}_{K}$ in $L^{2}(X, \mu ; \mathcal{Y})$. Since the probability measure $\mu$ is unknown, we require that the above property holds for any choice of $\mu$-compare with the definition of universal consistency for a learning algorithm [18]. Under the condition that the elements of $\mathcal{H}_{K}$ are continuous functions vanishing at infinity, we prove that universality of $\mathcal{H}_{K}$ is equivalent to require that $\mathcal{H}_{K}$ is dense in $\mathcal{C}_{0}(X ; \mathcal{Y})$, the Banach space of continuous functions vanishing at infinity with the uniform norm. If $X$ is compact and $\mathcal{H}=\mathbb{C}$, the density of $\mathcal{H}_{K}$ in $\mathcal{C}_{0}(X ; \mathcal{Y})$ is precisely the definition of universality given in [30, 32. For arbitrary $X$ and $\mathcal{Y}$, another definition of universality is suggested in [5] under the assumption that the elements of $\mathcal{H}_{K}$ are continuous functions. We show that this last notion is equivalent to require that $\mathcal{H}_{K}$ is dense in $L^{2}(X, \mu ; \mathcal{Y})$ for any probability measure $\mu$ with compact support, or that $\mathcal{H}_{K}$ is dense in $\mathcal{C}(X ; \mathcal{Y})$, the space of continuous functions with the compact-open topology. If $X$ is not compact, the two definitions of universality are not equivalent, as we show in two examples. To avoid confusion we refer to the second notion as compact-universality.

We characterize both universality and compact-universality in terms of the injectivity of the integral operator on $L^{2}(X, \mu ; \mathcal{Y})$ whose kernel is the reproducing kernel $K$. For compact-universal kernels, this result is presented in a slightly different form in [5] - compare Theorem 2 below with Theorem 11 of [5]. However, our statement of the theorem does not require a direct use of vector valued measures, our proof is simpler and it is based on the fact that any bounded linear functional $T$ on $\mathcal{C}_{0}(X ; \mathcal{Y})$ is of the form

$$
T(f)=\int_{X}\langle f(x), h(x)\rangle \mathrm{d} \mu(x),
$$

where $\mu$ is a probability measure and $h$ is a bounded measurable function from $X$ to $\mathcal{Y}$ - see Appendix A, Notice that, though in learning theory the main issue is the density of the RKHS $\mathcal{H}_{K}$ in $L^{2}(X, \mu ; \mathcal{Y})$, however, our results hold if, in the definition of universal kernels, we replace $L^{2}(X, \mu ; \mathcal{Y})$ with $L^{p}(X, \mu ; \mathcal{Y})$ for any $1 \leq p<\infty$. In particular, we show that $\mathcal{H}_{K}$ is dense in $\mathcal{C}_{0}(X ; \mathcal{Y})$ if and only if there exists $1 \leq p<\infty$ such that $\mathcal{H}_{K}$ is 
dense in $L^{p}(X, \mu ; \mathcal{Y})$ for any probability measure $\mu$. In that case, $\mathcal{H}_{K}$ is dense in $L^{q}(X, \mu ; \mathcal{Y})$ for any $1 \leq q<\infty$.

In the third part of the paper, under the assumption that $X$ is a group, Section 5 studies translation invariant reproducing kernels, that is, the kernels such that $K(x, t)=K_{e}\left(t^{-1} x\right)$ for some operator valued function $K_{e}: X \rightarrow$ $\mathcal{L}(\mathcal{Y})$ of completely positive type. In particular, we show that any translation invariant kernel is of the form

$$
K(x, t)=A \pi_{x^{-1} t} A^{*}
$$

for some unitary representation $\pi$ of $X$ acting on a Hilbert space $\mathcal{H}$, and a bounded operator $A: \mathcal{H} \rightarrow \mathcal{Y}$. If $X$ is an abelian group, SNAG theorem [16] provides a more explicit description of the reproducing kernel $K$, namely

$$
K(x, t)=\int_{\hat{X}} \chi(t-x) d Q(\chi)
$$

where $\hat{X}$ is the dual group and $Q$ is a positive operator valued measure on $\hat{X}$. The above equation is precisely the content of Bochner theorem for operator valued functions of positive type [2, 15]. In particular, we show that the corresponding RKHS $\mathcal{H}_{K}$ can be always realized as a closed subspace of $L^{2}(\hat{X}, \hat{\nu}, \mathcal{Y})$ where $\hat{\nu}$ is a suitable positive measure on $\hat{X}$. In this setting, we give a sufficient condition ensuring that a translation invariant kernel is universal. This condition is also necessary if $X$ is compact or $\mathcal{Y}=\mathbb{C}$. For scalar kernels and compact-universality this result is given in [22]. We end the paper by discussing in Section 6 the universality of some of the examples introduced in Section 3 ,

\section{Background}

In this section we set the main notations and we recall some basic facts about vector valued reproducing kernels.

\subsection{Notations and assumptions}

In the following we fix a locally compact second countable topological space $X$ and a complex separable Hilbert space $\mathcal{Y}$, whose norm and scalar product are denoted by $\|\cdot\|$ and $\langle\cdot, \cdot\rangle$ respectively. Local compactness of $X$ is needed in order to prove Theorem 7 in the appendix, which is at the root of Theorem 1 . The separability of $X$ and $\mathcal{Y}$ will avoid some problems in measure theory. All these assumptions are always satisfied in learning theory. 
We denote by $\mathcal{F}(X ; \mathcal{Y})$ the vector space of functions $f: X \rightarrow \mathcal{Y}$, by $\mathcal{C}(X ; \mathcal{Y})$ the subspace of continuous functions, and by $\mathcal{C}_{0}(X ; \mathcal{Y})$ the subspace of continuous functions vanishing at infinity. If $\mathcal{Y}=\mathbb{C}$, we set $\mathcal{C}(X)=$ $\mathcal{C}(X ; \mathbb{C})$ and $\mathcal{C}_{0}(X)=\mathcal{C}_{0}(X, \mathbb{C})$. If $X$ is compact, $\mathcal{C}_{0}(X ; \mathcal{Y})=\mathcal{C}(X ; \mathcal{Y})$.

We regard $\mathcal{C}(X ; \mathcal{Y})$ as a locally convex topological vector space by endowing it with the compact-open topology ${ }^{1}$ and $\mathcal{C}_{0}(X ; \mathcal{Y})$ as a Banach space with respect to the uniform norm $\|f\|_{\infty}=\max _{x \in X}\|f(x)\|$.

Let $\mathcal{B}(X)$ be the Borel $\sigma$-algebra of $X$. By a measure on $X$ we mean a $\sigma$-additive map $\mu: \mathcal{B}(X) \longrightarrow[0,+\infty]$ which is finite on compact set: 2 . We say that $\mu$ is a probability measure if $\mu(X)=1$. For $1 \leq p<\infty$, $L^{p}(X, \mu ; \mathcal{Y})$ denotes the Banach space of (equivalence classes of) measurable functions $f: X \rightarrow \mathcal{Y}$ such that $\|f\|^{p}$ is $\mu$-integrable, with norm $\|f\|_{p}=\left(\int_{X}\|f(x)\|^{p} \mathrm{~d} \mu(x)\right)^{1 / p}$. If $p=2$ we denote the scalar product in $L^{2}(X, \mu, \mathcal{Y})$ by $\langle\cdot, \cdot\rangle_{2}$. For $p=\infty, L^{\infty}(X, \mu ; \mathcal{Y})$ is the Banach space of $\mu$ essentially bounded measurable functions $f: X \rightarrow \mathcal{Y}$ with norm $\|f\|_{\mu, \infty}=$ $\mu-\operatorname{ess} \sup _{x \in X}\|f(x)\|$.

If $\mu$ is a probability measure, clearly

$$
\mathcal{C}_{0}(X ; \mathcal{Y}) \subset L^{p}(X, \mu ; \mathcal{Y}) \subset L^{q}(X, \mu ; \mathcal{Y})
$$

for all $1 \leq q<p \leq \infty$, each inclusion being continuous. Moreover, since $X$ is locally compact and second countable, $\mathcal{C}_{0}(X ; \mathcal{Y})$ is dense in $L^{p}(X, \mu ; \mathcal{Y})$ for any $1 \leq p<\infty$.

If $\mathcal{H}$ is an arbitrary (complex) Hilbert space we denote its scalar product by $\langle\cdot, \cdot\rangle_{\mathcal{H}}$ and its norm by $\|\cdot\|_{\mathcal{H}}$. When $\mathcal{H}^{\prime}$ is another Hilbert space, we denote by $\mathcal{L}\left(\mathcal{H} ; \mathcal{H}^{\prime}\right)$ the Banach space of bounded operators from $\mathcal{H}$ to $\mathcal{H}^{\prime}$ endowed with the uniform norm. In the case $\mathcal{H}=\mathcal{H}^{\prime}$, we set $\mathcal{L}(\mathcal{H})=\mathcal{L}(\mathcal{H} ; \mathcal{H})$.

Given $w_{1}, w_{2} \in \mathcal{H}$, we let $w_{1} \otimes w_{2}$ be the rank one operator

$$
\left(w_{1} \otimes \overline{w_{2}}\right) v=\left\langle v, w_{2}\right\rangle_{\mathcal{H}} w_{1} \quad v \in \mathcal{H} .
$$

\subsection{Vector valued reproducing kernels}

We briefly recall the main properties of vector valued reproducing kernel Hilbert spaces. Given $X$ and $\mathcal{Y}$ as above, a map $K: X \times X \longrightarrow \mathcal{L}(\mathcal{Y})$ is

\footnotetext{
${ }^{1}$ This is the topology of uniform convergence on compact subsets defined by the family of seminorms $\|f\|_{Z}=\max _{x \in Z}\|f(x)\|$ for $Z$ varying over the compact subsets in $X$.

${ }^{2}$ Since $X$ is locally compact second countable, then $\mu$ is both inner and outer regular.

${ }^{3}$ Since $\mathcal{Y}$ is separable, measurability is equivalent to the fact that $\langle f(\cdot), y\rangle$ is measurable for all $y \in \mathcal{Y}$.
} 
called a $\mathcal{Y}$-reproducing kernel if

$$
\sum_{i, j=1}^{N}\left\langle K\left(x_{i}, x_{j}\right) y_{j}, y_{i}\right\rangle \geq 0
$$

for any $x_{1}, \ldots, x_{N}$ in $X, y_{1}, \ldots, y_{N}$ in $\mathcal{Y}$ and $N \geq 1$. Given $x \in X, K_{x}: \mathcal{Y} \rightarrow$ $\mathcal{F}(X ; \mathcal{Y})$ denotes the linear operator whose action on a vector $y \in \mathcal{Y}$ is the function $K_{x} y \in \mathcal{F}(X ; \mathcal{Y})$ defined by

$$
\left(K_{x} y\right)(t)=K(t, x) y \quad t \in X .
$$

Given a $\mathcal{Y}$-reproducing kernel $K$, there is a unique Hilbert space $\mathcal{H}_{K} \subset \mathcal{F}(X ; \mathcal{Y})$ satisfying

$$
\begin{array}{ll}
K_{x} \in \mathcal{L}\left(\mathcal{Y}, \mathcal{H}_{K}\right) & x \in X \\
f(x)=K_{x}^{*} f & x \in X, f \in \mathcal{H}_{K},
\end{array}
$$

where $K_{x}^{*}: \mathcal{H}_{K} \rightarrow \mathcal{Y}$ is the adjoint of $K_{x}$, see Proposition 2.1 of [6]. The space $\mathcal{H}_{K}$ is called the reproducing kernel Hilbert space associated with $K$, the corresponding scalar product and norm are denoted by $\langle\cdot, \cdot\rangle_{K}$ and $\|\cdot\|_{K}$, respectively. As a consequence of (3), we have that

$$
\begin{aligned}
& K(x, t)=K_{x}^{*} K_{t} \quad x, t \in X \\
& \mathcal{H}_{K}=\overline{\operatorname{span}}\left\{K_{x} y \mid x \in X, y \in \mathcal{Y}\right\} .
\end{aligned}
$$

As discussed in the introduction, the space $\mathcal{H}_{K}$ can be realized as a closed subspace of some arbitrary Hilbert space by means of a suitable feature map, as shown by the next result, see Proposition 2.4 of [6].

Proposition 1. Let $\mathcal{H}$ be a Hilbert space and $\gamma: X \longrightarrow \mathcal{B}(\mathcal{Y} ; \mathcal{H})$. Then the operator $W: \mathcal{H} \longrightarrow \mathcal{F}(X ; \mathcal{Y})$ defined by

$$
(W u)(x)=\gamma_{x}^{*} u, \quad u \in \mathcal{H}, x \in X,
$$

is a partial isometry from $\mathcal{H}$ onto the reproducing kernel Hilbert space $\mathcal{H}_{K}$ with reproducing kernel

$$
K(x, t)=\gamma_{x}^{*} \gamma_{t}, \quad x, t \in X .
$$

Moreover, $W^{*} W$ is the orthogonal projection onto

$$
\operatorname{ker} W^{\perp}=\overline{\operatorname{span}}\left\{\gamma_{x} y \mid x \in X, y \in \mathcal{Y}\right\} \text {, }
$$

and

$$
\|f\|_{K}=\inf \left\{\|u\|_{\mathcal{H}} \mid u \in \mathcal{H}, W u=f\right\} .
$$


The map $\gamma$ is usually called the feature map, $W$ the feature operator and $\mathcal{H}$ the feature space. Since $W$ is an isometry from ker $W^{\perp}$ onto $\mathcal{H}_{K}$, the map $W$ allows us to identify $\mathcal{H}_{K}$ with the closed subspace ker $W^{\perp}$ of $\mathcal{H}$. With a mild abuse of notation, we say that $\mathcal{H}_{K}$ is embedded into $\mathcal{H}$ by means of the feature operator $W$.

Comparing (41) with (3), we notice that any RKHS $\mathcal{H}_{K}$ admits a trivial feature map, namely $\gamma_{x}=K_{x}$. In this case the feature operator is the identity. Conversely, if $\mathcal{H}$ is a Hilbert space of functions from $X$ to $\mathcal{Y}$ such that $\|f\| \leq$ $C_{x}\|f\|_{\mathcal{H}}$ for some positive constant $C_{x}$, then there exists a bounded operator $\gamma_{x}: \mathcal{Y} \rightarrow \mathcal{H}$ such that $f(x)=\gamma_{x}^{*} f$. Hence, the above proposition implies that $\mathcal{H}$ is a RKHS with kernel given by (5) and that the feature operator is the identity.

\subsection{Mercer and $\mathcal{C}_{0}$-kernels}

In this paper, we mainly focus on reproducing kernel Hilbert spaces, whose elements are continuous functions. In particular we study the following two classes of reproducing kernels.

Definition 1. A reproducing kernel $K: X \times X \rightarrow \mathcal{L}(\mathcal{Y})$ is called

(i) Mercer provided that $\mathcal{H}_{K}$ is a subspace of $\mathcal{C}(X ; \mathcal{Y})$;

(ii) $\mathcal{C}_{0}$ provided that $\mathcal{H}_{K}$ is a subspace of $\mathcal{C}_{0}(X ; \mathcal{Y})$.

The choice of $\mathcal{C}(X ; \mathcal{Y})$ and $\mathcal{C}_{0}(X ; \mathcal{Y})$ is motivated in Section 4 where we discuss the universality problem.

The following proposition directly characterizes Mercer and $\mathcal{C}_{0^{-}}$kernels in terms of properties of the kernels.

Proposition 2. Let $K$ be a reproducing kernel.

(i) The kernel $K$ is Mercer iff the function $x \longmapsto\|K(x, x)\|$ is locally bounded and $K_{x} y \in \mathcal{C}(X ; \mathcal{Y})$ for all $x \in X$ and $y \in \mathcal{Y}$.

(ii) The kernel $K$ is $\mathcal{C}_{0}$ iff the function $x \longmapsto\|K(x, x)\|$ is bounded and $K_{x} y \in \mathcal{C}_{0}(X ; \mathcal{Y})$ for all $x \in X$ and $y \in \mathcal{Y}$.

If $K$ is a Mercer kernel, the inclusion $\mathcal{H}_{K} \hookrightarrow \mathcal{C}(X ; \mathcal{Y})$ is continuous. If $K$ is a $\mathcal{C}_{0}$-kernel the inclusion $\mathcal{H}_{K} \hookrightarrow \mathcal{C}_{0}(X ; \mathcal{Y})$ is continuous. In both cases, the space $\mathcal{H}_{K}$ is separable. 
Proof. We prove only (ii), since the other proof is similar - see Proposition 5.1 of [6]. If $\mathcal{H}_{K} \subset \mathcal{C}_{0}(X ; \mathcal{Y})$, it is clear that $K_{x} y$ is an element of $\mathcal{C}_{0}(X ; \mathcal{Y})$. Moreover, since $\left\|K_{x}^{*} f\right\|=\|f(x)\| \leq\|f\|_{\infty} \forall f \in \mathcal{H}_{K}$, by the principle of uniform boundedness there exists $M<\infty$ such that $\left\|K_{x}^{*}\right\| \leq M$ for all $x$. Therefore, $\|K(x, x)\|=\left\|K_{x}^{*}\right\|^{2} \leq M^{2}$ for all $x$.

Conversely, assume that the function $x \longmapsto\|K(x, x)\|$ is bounded and $K_{x} y \in$ $\mathcal{C}_{0}(X ; \mathcal{Y})$. Given $f \in \mathcal{H}_{K}$, we have

$$
\|f(x)\| \leq\|f\|_{K}\|K(x, x)\|^{1 / 2} \leq M\|f\|_{K} .
$$

In particular, convergence in $\mathcal{H}_{K}$ implies uniform convergence, so that the closure (in $\mathcal{H}_{K}$ ) of the linear span of $\left\{K_{x} y \mid x \in X, y \in \mathcal{Y}\right\}$ is contained in $\mathcal{C}_{0}(X ; \mathcal{Y})$, i.e. $\mathcal{H}_{K} \subseteq \mathcal{C}_{0}(X ; \mathcal{Y})$.

The continuity of the inclusion of $\mathcal{H}_{K}$ in $\mathcal{C}_{0}(X ; \mathcal{Y})$ follows from $\|f\|_{\infty} \leq$ $M\|f\|_{\mathcal{H}_{K}}$. Finally $\mathcal{H}_{K}$ is separable by Corollary 5.2 of [6].

If $\mathcal{H}_{K}$ is defined by means of a feature map $\gamma$, the above characterization can be expressed in terms of $\gamma$, as shown by the following result.

Corollary 1. With the notations of Proposition 1 the following conditions are equivalent.

(a) The kernel $K$ is Mercer $\left[\right.$ resp. $\left.\mathcal{C}_{0}\right]$.

(b) There is a total set $\mathcal{S}$ in $\mathcal{H}$ such that $W(\mathcal{S}) \subset \mathcal{C}(X ; \mathcal{Y})[$ resp. $W(\mathcal{S}) \subset$ $\left.\mathcal{C}_{0}(X ; \mathcal{Y})\right]$ and the function $x \longmapsto\left\|\gamma_{x}\right\|$ is locally bounded [resp. bounded].

Proof. We give the proof only in the case of a $\mathcal{C}_{0}$-kernel, the other case being simpler. Suppose hence (a) holds true, i.e. $\mathcal{H}_{K} \subset \mathcal{C}_{0}$, then $W(\mathcal{S}) \subset$ ran $W=$ $\mathcal{H}_{K} \subset \mathcal{C}_{0}(X ; \mathcal{Y})$ for all subset $\mathcal{S}$ of $\mathcal{H}$. Moreover, $\left\|\gamma_{x}\right\|^{2}=\|K(x, x)\| \leq M$ by item (ii) of Proposition 2, Conversely, if condition (b) holds, we have that for all $x \in X$ and $u \in \mathcal{H}$

$\|(W u)(x)\|=\left\|K_{x}^{*}(W u)\right\| \leq\left\|K_{x}^{*}\right\|\|W\|\|u\|_{\mathcal{H}} \leq\|K(x, x)\|^{\frac{1}{2}}\|u\|_{\mathcal{H}} \leq M^{\frac{1}{2}}\|u\|_{\mathcal{H}}$,

where $\|W\| \leq 1$ being $W$ a partial isometry. Then $W$ maps $\mathcal{H}$ into the space of bounded functions and $W$ is continuous from $\mathcal{H}$ onto $\mathcal{H}_{K}$ endowed with the uniform norm. Since $W(\mathcal{S}) \subset \mathcal{C}_{0}(X ; \mathcal{Y})$ and $\mathcal{C}_{0}(X ; \mathcal{Y})$ is complete, then $\mathcal{H}_{K} \subset \mathcal{C}_{0}(X ; \mathcal{Y})$. 


\subsection{Mercer theorem}

For a Mercer kernel $K$, there is a canonical feature map, based on Mercer theorem, which relates the spectral properties of the integral operator with kernel $K$, and the structure of the corresponding reproducing kernel Hilbert space. This result will be also used in the examples.

To state this result for vector valued reproducing kernels, we need some preliminary facts. First of all, if $K$ is a Mercer kernel and $\mu$ is a probability measure on $X$, the space $\mathcal{H}_{K}$ is a subspace of $L^{2}(X, \mu ; \mathcal{Y})$, provided that $\|K(x, x)\|$ is bounded on the support of $\mu$. This last condition is always satisfied if $K$ is a $\mathcal{C}_{0}$-kernel or if $\mu$ has compact support. If $\mathcal{H}_{K}$ is a subspace of $L^{2}(X, \mu ; \mathcal{Y})$, we denote the canonical inclusion by

$$
i_{\mu}: \mathcal{H}_{K} \hookrightarrow L^{2}(X, \mu ; \mathcal{Y})
$$

Next lemma states some properties of $i_{\mu}$ and its proof is a consequence of Propositions 3.3, 4.4 and 4.8 of [6].

Proposition 3. Let $K$ be a Mercer kernel and $\mu$ a probability measure such that $K$ is bounded on the support of $\mu$. The inclusion $i_{\mu}$ is a bounded operator, its adjoint $i_{\mu}^{*}: L^{2}(X, \mu ; \mathcal{Y}) \longrightarrow \mathcal{H}_{K}$ is given by

$$
\left(i_{\mu}^{*} f\right)(x)=\int_{X} K(x, t) f(t) \mathrm{d} \mu(t),
$$

where the integral converges in norm, and the composition $i_{\mu} i_{\mu}^{*}=L_{\mu}$ is the integral operator on $L^{2}(X, \mu ; \mathcal{Y})$ with kernel $K$

$$
\left(L_{\mu} f\right)(x)=\int_{X} K(x, t) f(t) \mathrm{d} \mu(t) .
$$

In particular, if $K(x, x)$ is a compact operator for all $x \in X$, then $L_{K}$ is a compact operator.

The fact that $L_{K}$ is a compact operator implies that there is a family $\left(f_{i}\right)_{i \in I}$ of eigenvectors in $\mathcal{C}(X ; \mathcal{Y})$ and a family $\left(\sigma_{i}\right)_{i \in I}$ of eigenvalues in $] 0, \infty[$ such that $\left(f_{i}\right)_{i \in I}$ is an orthonormal basis of $\operatorname{ker} L_{\mu}{ }^{\perp}=\overline{\operatorname{ran} L_{\mu}}$ and

$$
L_{\mu} f_{i}=\sigma_{i} f_{i}
$$

With this notation we are ready to state Mercer Theorem for vector valued kernels. Its proof is consequence of Proposition 6.1 and Theorem 6.3 of [6]. 
Proposition 4. Let $\mu$ be a probability measure with supp $\mu=X$. Suppose $K$ is a Mercer kernel such that $\sup _{x \in X}\|K(x, x)\|<\infty$, and $K(x, x)$ is a compact operator $\forall x \in X$. With the notation of (6) , we have that

$$
\begin{aligned}
& \mathcal{H}_{K}=\left\{f \in \mathcal{C}(X ; \mathcal{Y}) \cap \operatorname{ker} L_{\mu}{ }^{\perp} \mid \sum_{i \in I} \frac{\left|\left\langle f, f_{i}\right\rangle_{2}\right|^{2}}{\sigma_{i}}<\infty\right\} \\
& \langle f, g\rangle_{K}=\sum_{i \in I} \frac{\left\langle f, f_{i}\right\rangle_{2}\left\langle f_{i}, g\right\rangle_{2}}{\sigma_{i}} \\
& K(x, t)=\sum_{i \in I} \sigma_{i} f_{i}(x) \otimes \overline{f_{i}(t)}
\end{aligned}
$$

where the last series converges in the strong operator topology of $\mathcal{L}(\mathcal{Y})$.

Equations (77) and (8) imply that $\left(\sqrt{\sigma_{i}} f_{i}\right)_{i \in I}$ is an orthonormal basis in $\mathcal{H}_{K}$. In particular the vectors $\sqrt{\sigma_{i}} f_{i}$ are $\ell_{2}$-linearly independent in $\mathcal{F}(X ; \mathcal{Y})$, namely, if $\left(c_{i}\right)_{i \in I}$ is a family such that $\sum_{i \in I}\left|c_{i}\right|^{2}<\infty$ and $\sum_{i \in I} c_{i} \sqrt{\sigma_{i}} f_{i}(x)=$ 0 for all $x \in X$, then $c_{i}=0$ for all $i \in I$.

As said at the beginning of Section 2.4. Proposition 4 gives a feature operator, which is often used in learning theory.

Example 1. With the assumptions and notations of Proposition 4, the reproducing kernel Hilbert space $\mathcal{H}_{K}$ is unitarily equivalent to ker $L_{\mu}{ }^{+}=\overline{\operatorname{ran} L_{\mu}}$ by means of the feature operator

$$
(W f)(x)=\sum_{i \in I} \sqrt{\sigma_{i}} f_{i}(x)\left\langle f, f_{i}\right\rangle_{2}=\left(L_{\mu}^{\frac{1}{2}} f\right)(x), \quad f \in L^{2}(X, \mu ; \mathcal{Y}) .
$$

Proof. Given $x \in X$, define

$$
\gamma_{x}: \mathcal{Y} \rightarrow L^{2}(X, \mu ; \mathcal{Y}) \quad \gamma_{x} y=\sum_{i \in I} \sqrt{\sigma_{i}}\left\langle y, f_{i}(x)\right\rangle f_{i}
$$

which is well defined since $\left(f_{i}\right)_{i \in I}$ is orthonormal family of continuous functions and (9) ensures that $\sum_{i \in I} \sigma_{i}\left|\left\langle y, f_{i}(x)\right\rangle\right|^{2}<\infty$. Using (9) again, one checks that $\gamma_{x}^{*} \gamma_{t}=K(x, t)$. The fact that feature operator is given by (10) is clear by definition of $\gamma_{x}$. Since $\operatorname{ker} W=\operatorname{ker} L_{\mu}, W$ is a unitary operator from $\operatorname{ker} L_{\mu}{ }^{\perp}$ onto $\mathcal{H}_{K}$.

\subsection{Trivial examples}

We give two examples of trivial vector valued kernels. 
Example 2. Let $B \in \mathcal{L}(\mathcal{Y})$ be a positive operator and define $K(x, t)=B$ for all $x, t \in X$, then $K$ is a $\mathcal{Y}$-reproducing kernel, $\mathcal{H}_{K}$ is unitarily equivalent to $\operatorname{ker} B^{\perp}=\overline{\operatorname{ran} B}$ by means of the feature operator

$$
(W y)(x)=B^{\frac{1}{2}} y \quad x \in X, y \in \operatorname{ker} B^{\perp} .
$$

The kernel $K$ is of Mercer type and it is a $\mathcal{C}_{0}$-kernel if and only if $X$ is compact.

Proof. Apply Proposition 1 with $\mathcal{H}=\operatorname{ker} B^{\perp}$ and $\gamma_{x}=B^{\frac{1}{2}}$. Since $B$ is injective on $\mathcal{H}$, then $W$ is unitary. The claims about the continuity are clear.

Example 3. Let $f: X \rightarrow \mathcal{Y}, f \neq 0$. Define $K(x, t)=f(x) \otimes \overline{f(t)}$, then $K$ is a reproducing kernel, $\mathcal{H}_{K}$ is unitarily equivalent to $\mathbb{C}$ by means of the feature operator

$$
(W c)(x)=c f(x) \quad x \in X, c \in \mathbb{C} .
$$

In particular $K$ is Mercer $\left[\right.$ resp. $\left.\mathcal{C}_{0}\right]$ if and only if $f \in \mathcal{C}(X ; \mathcal{Y})[$ resp. $f \in$ $\left.\mathcal{C}_{0}(X ; \mathcal{Y})\right]$

Proof. Apply Proposition 1 with $\mathcal{H}=\mathbb{C}$ and $\gamma_{x} y=\langle y, f(x)\rangle$. Since $f \neq 0$, $W$ is injective. The characterization about Mercer and $\mathcal{C}_{0}$ is trivial.

\section{Operations with kernels}

In this section we characterize reproducing kernel Hilbert spaces whose kernel is defined by algebraic operations, like sum, product and composition. Most of the results are well known for scalar kernels, whereas for vector valued kernels they are consequences of the theory developed in [27] in a more general context. We provide a direct and simple proof of these results, based on the use of suitable feature maps. In some cases, our approach can be of interest also in the scalar case, like, for example, in proving Schur lemma about the product of kernels.

As an application, we present a large supply of examples of vector valued reproducing kernels and, for most of them, we realize the corresponding RKHS by elegant and simple structures. This characterization will be used to analyze some learning algorithm, like regularized least-squares, in the vector valued setting. 


\subsection{Sum of kernels}

The following result extends to vector valued kernels the relation between sum of kernels and sum of the corresponding reproducing kernel Hilbert spaces.

Proposition 5. Denote by $I$ a countable set and let $\left(K^{i}\right)_{i \in I}$ be a family of $\mathcal{Y}$-reproducing kernels such that

$$
\sum_{i \in I}\left\langle K^{i}(x, x) y, y\right\rangle<\infty \quad \forall y \in \mathcal{Y} \text { and } \forall x \in X
$$

Given $x, t \in X$ the series $\sum_{i \in I} K^{i}(x, t)$ converges to a bounded operator $K(x, t)$ in the strong operator topology, and the map $K: X \times X \rightarrow \mathcal{L}(\mathcal{Y})$ defined by

$$
K(x, t) y=\sum_{i \in I} K^{i}(x, t) y
$$

is a $\mathcal{Y}$-reproducing kernel. The corresponding space $\mathcal{H}_{K}$ is embedded in $\bigoplus_{i \in I} \mathcal{H}_{K^{i}}$ by means of the feature operator

$$
W(f)(x)=\sum_{i \in I} f_{i}(x) \quad \text { where } f=\oplus_{i \in I} f_{i}
$$

where the sum converges in norm.

Moreover, if each $K^{i}$ is a Mercer kernel [resp. $\mathcal{C}_{0}$-kernel] and $x \mapsto \sum_{i \in I}\left\|K^{i}(x, x)\right\|$ is locally bounded [resp. bounded], then $K$ is Mercer [resp. $\mathcal{C}_{0}$ ].

Proof. We apply Proposition 1, Letting $\mathcal{H}=\bigoplus_{i \in I} \mathcal{H}_{K^{i}}$, we regard each $\mathcal{H}_{K^{i}}$ as a closed subspace of $\mathcal{H}$ so that any two of them are orthogonal. Given $x \in X$, we define the bounded operator $\gamma_{x}: \mathcal{Y} \rightarrow \mathcal{H}$ by $\gamma_{x}=\sum_{i \in I} K_{x}^{i}$, where the series converges in the strong operator topology since, given $y \in \mathcal{Y}$,

$$
\sum_{i \in I}\left\|K_{x}^{i} y\right\|_{K^{i}}^{2}=\sum_{i \in I}\left\langle K^{i}(x, x) y, y\right\rangle<\infty
$$

by assumption, see [7]. Given $i \in I$ and $f_{i} \in \mathcal{H}_{K^{i}}$, then

$$
\left\langle\gamma_{x}^{*} f_{i}, y\right\rangle=\left\langle f_{i}, K_{x}^{i} y\right\rangle_{K^{i}}=\left\langle f_{i}(x), y\right\rangle
$$

by reproducing property (3), so that $\gamma_{x}^{*} f_{i}=f_{i}(x)$. Since $\gamma_{x}^{*}$ is continuous, for any $f=\oplus_{i \in I} f_{i}$,

$$
(W f)(x)=\gamma_{x}^{*} f=\sum_{i \in I} \gamma_{x}^{*} f_{i}=\sum_{i \in I} f_{i}(x)
$$


where the series converges in norm.

Finally, $K(x, t) y=\gamma_{x}^{*} \gamma_{t} y=\sum_{i \in I}\left(\gamma_{t} y\right)_{i}(x)=\sum_{i \in I} K^{i}(x, t) y$, that is $K(x, t)=$ $\sum_{i \in I} K^{i}(x, t)$ in the strong operator topology.

The second part is a consequence of Corollary 1 with $\mathcal{S}=\bigcup_{i \in I} \mathcal{H}_{K^{i}}$.

As an application, we have the following example.

Example 4. Let $\left(f_{i}\right)_{\in I}$ a countable family of functions $f_{i}: X \rightarrow \mathcal{Y}$ such that $\sum_{i \in I}\left|\left\langle f_{i}(x), y\right\rangle\right|^{2}$ is finite for all $x \in X$ and $y \in Y$. Define $K: X \times X \rightarrow$ $\mathcal{L}(\mathcal{Y})$ as

$$
K(x, t)=\sum_{i \in I} f_{i}(x) \otimes \overline{f_{i}(t)} .
$$

Then, the sum converges in the strong operator topology, $K$ is a reproducing kernel and

$$
\mathcal{H}_{K}=\left\{\left.f \in \mathcal{F}(X ; \mathcal{Y})\left|f(x)=\sum_{i \in I} c_{i} f_{i}(x), \sum_{i \in I}\right| c_{i}\right|^{2}<\infty\right\} .
$$

In particular $\left(f_{i}\right)_{i \in I}$ is a normalized tight frame in $\mathcal{H}_{K}$. It is an orthonormal basis if and only if $\left(f_{i}\right)_{i \in I}$ is $\ell_{2}$-linearly independent in $\mathcal{F}(X ; \mathcal{Y})$.

Proof. Apply Proposition 5, with $K^{i}(x, t)=f_{i}(x) \otimes \overline{f_{i}(t)}$, observing that $\mathcal{H}_{K^{i}}=\mathbb{C}$ by Example 3, so that $\oplus_{i \in I} \mathcal{H}_{K_{i}} \simeq \ell_{2}$. The feature operator is explicitly given by

$$
W(c)(x)=\sum_{i \in I} c_{i} f_{i}(x) \quad \text { where } c=\left(c_{i}\right)_{i \in I}, \sum_{i \in I}\left|c_{i}\right|^{2}<\infty,
$$

so that (11) is clear. If $\left(e_{i}\right)_{i \in I}$ is the canonical orthonormal basis of $\ell_{2}$, then $W e_{i}=f_{i}$ and, for any $f \in \mathcal{H}_{K}$,

$$
\|f\|_{K}^{2}=\left\|W^{*} f\right\|_{\ell_{2}}^{2}=\sum_{i}\left|\left\langle W^{*} f, e_{i}\right\rangle_{\ell_{2}}\right|^{2}=\sum_{i}\left|\left\langle f, f_{i}\right\rangle_{K}\right|^{2},
$$

i.e. $\left(f_{i}\right)_{i \in I}$ is a normalized tight frame in $\mathcal{H}_{K}$. Clearly, it is an orthonormal basis if and only if $W$ is unitary, i.e. $\mathrm{W}$ is injective. This is precisely the condition that $\left(f_{i}\right)_{i \in I}$ is $\ell_{2}$-linearly independent in $\mathcal{F}(X ; \mathcal{Y})$.

Proposition 4 shows that any RKHS with a bounded compact Mercer kernel can be realized as in the above example, where the functions $f_{i}$ are the eigenfunctions (with $\left\|f_{i}\right\|_{2}^{2}=\sigma_{i}$ ) of the integral operator $L_{\mu}$ with eigenvalues $\sigma_{i}>0$, and $\mu$ is any probability measure with supp $\mu=X$, see (6). 


\subsection{Composition with maps}

We now describe the reproducing kernel Hilbert spaces whose kernel is defined in terms of a mother kernel and suitable maps acting either on the input space $X$ or on the output space $\mathcal{Y}$. The following result characterizes the action of a bounded operator on $\mathcal{Y}$.

Proposition 6. Let $K$ be a $\mathcal{Y}$-reproducing kernel. Let $\mathcal{Y}^{\prime}$ be another Hilbert space and $w: \mathcal{Y} \rightarrow \mathcal{Y}^{\prime}$ be a bounded operator. Define

$$
K_{w}: X \times X \rightarrow \mathcal{L}\left(\mathcal{Y}^{\prime}\right) \quad K_{w}(x, t)=w K(x, t) w^{*},
$$

then $K_{w}$ is a $\mathcal{Y}^{\prime}$ reproducing kernel and $\mathcal{H}_{K_{w}}$ is embedded in $\mathcal{H}_{K}$ by means of

$$
W: \mathcal{H}_{K} \longrightarrow \mathcal{H}_{K_{w}}, \quad(W f)(x)=w f(x) \quad x \in X .
$$

If $w$ is injective, $\mathcal{H}_{K_{w}}$ is unitarily equivalent to $\mathcal{H}_{K}$. Moreover, if $K$ is Mercer [resp. $\left.\mathcal{C}_{0}\right]$, then $K_{w}$ is Mercer [resp. $\left.\mathcal{C}_{0}\right]$.

Proof. Let $\gamma_{x}: \mathcal{Y}^{\prime} \rightarrow \mathcal{H}_{K}, \gamma_{x}=K_{x} w^{*}$ and apply Proposition 1 with $\mathcal{H}=\mathcal{H}_{K}$. The feature operator from $\mathcal{H}_{K}$ onto $\mathcal{H}_{K_{w}}$ is explicitly given by $(W f)(x)=$ $\gamma_{x}^{*} f=w f(x)$. If $w$ is injective, then $W$ is unitary. The second claim is evident.

We now study the action of an arbitrary map on $X$.

Proposition 7. Let $K$ be a $\mathcal{Y}$-reproducing kernel on $X$. Let $T$ be another locally compact second countable topological space, and $\Psi: T \rightarrow X$. Define

$$
K_{\Psi}: T \times T \rightarrow \mathcal{L}(\mathcal{Y}) \quad K_{\Psi}\left(t_{1}, t_{2}\right)=K\left(\Psi\left(t_{1}\right), \Psi\left(t_{2}\right)\right) \quad t_{1}, t_{2} \in T .
$$

Then $K_{\Psi}$ is a $\mathcal{Y}$-reproducing kernel on $T$, the space $\mathcal{H}_{K_{\Psi}}$ is unitarily equivalent to

$$
\overline{\operatorname{span}}\left\{K_{x} y \mid x \in \operatorname{ran} \Psi\right\}=\left\{f \in \mathcal{H}_{K} \mid f(x)=0 \forall x \in \operatorname{ran} \Psi\right\}^{\perp}
$$

by means of the feature operator

$$
W: \mathcal{H}_{K} \longrightarrow \mathcal{H}_{K_{\Psi}} \quad W(f)(t)=f(\Psi(t)) \quad f \in \mathcal{H}_{K}, t \in T .
$$

If $K$ is a Mercer kernel and $\Psi$ is continuous, then $K_{\Psi}$ is Mercer. If $K$ is a $\mathcal{C}_{0}$-kernel and $\Psi$ is continuous and proper, then $K_{\Psi}$ is $\mathcal{C}_{0}$.

Proof. Apply Proposition [1 with $\mathcal{H}=\mathcal{H}_{K}$ and, for any $t \in T, \gamma_{t}=K_{\Psi(t)}$, observing that ker $W=\left\{f \in \mathcal{H}_{K} \mid f(x)=0 \forall x \in \operatorname{ran} \Psi\right\}$.

The claims about Mercer and $\mathcal{C}_{0}$-kernels are clear. 
In the above proposition observe that ker $W^{\perp}$ can be identified with the quotient space $\mathcal{H}_{K} /$ ker $W$, so that one has also the natural identification

$$
\mathcal{H}_{K_{\Psi}} \simeq\left\{f_{\mid \operatorname{ran} \Psi} \mid f \in \mathcal{H}_{K}\right\}
$$

where, the r.h.s. is endowed with the norm

$$
\left\|f_{\mid \operatorname{ran} \Psi}\right\|=\inf \left\{\|g\|_{K} \mid g \in \mathcal{H}_{K}, g_{\mid \operatorname{ran} \Psi}=f_{\mid \operatorname{ran} \Psi}\right\}
$$

As a consequence, we describe the relation between a kernel and its restriction to a subset.

Corollary 2. Let $X_{0}$ be a subset of $X$. Let $K_{X_{0}}$ be the restriction of $K$ to $X_{0} \times X_{0}$, then

$$
\mathcal{H}_{K_{X_{0}}}=\left\{f_{\left.\right|_{X_{0}}}: f \in \mathcal{H}_{K}\right\}
$$

If $K$ is Mercer and $X_{0}$ is locally closed, then $K_{X_{0}}$ is Mercer. If $K$ is $\mathcal{C}_{0}$ and $X_{0}$ is closed, then $K_{X_{0}}$ is $\mathcal{C}_{0}$.

Proof. Apply Proposition 7 and identification (12), with $\Psi$ the canonical inclusion of $X_{0}$ in $X$.

We end this part by describing the reproducing kernel Hilbert space associated with the kernel proposed in [5].

Proposition 8. Let $\kappa$ be a scalar reproducing kernel on $X$. Let $T$ be another locally compact second countable topological space. Let $\Psi_{1}, \ldots, \Psi_{m}$ be functions from $T$ to $X$ and define $K\left(t_{1}, t_{2}\right)$ as the $m \times m$-matrix

$$
K\left(t_{1}, t_{2}\right)_{i j}=\kappa\left(\Psi_{i}\left(t_{1}\right), \Psi_{j}\left(t_{2}\right)\right) \quad i, j=1, \ldots, m, t_{1}, t_{2} \in T .
$$

Then $K$ is a $\mathbb{C}^{m}$-reproducing kernel on $T$, the space $\mathcal{H}_{K}$ is embedded in $\mathcal{H}_{\kappa}$ by means of the feature operator

$$
W: \mathcal{H}_{\kappa} \longrightarrow \mathcal{H}_{K} \quad(W(\varphi)(t))_{i}=\varphi\left(\Psi_{i}(t)\right) \quad \varphi \in \mathcal{H}_{\kappa}, t \in T .
$$

If one of $\Psi_{1}, \ldots, \Psi_{m}$ is surjective, then $W$ is unitary.

Proof. Apply Proposition 1 with $\mathcal{H}=\mathcal{H}_{\kappa}$ and $\gamma_{t}: \mathbb{C}^{m} \rightarrow \mathcal{H}_{\kappa}$,

$$
\gamma_{t}\left(y_{1}, \ldots, y_{m}\right)=\sum_{i=1}^{m} y_{i} \kappa_{\Psi_{i}(t)},
$$

so that $\gamma_{t}^{*}(\varphi)_{i}=\varphi\left(\Psi_{i}(t)\right)$.

If $\Psi_{i}$ is surjective for some index $i=1, \ldots, m$, the condition $\varphi\left(\Psi_{i}(t)\right)=0$ for all $t \in T$ implies that $\varphi(x)=0$ for all $x \in X$, that is, $\varphi=0$. Hence $W$ is injective and, hence, unitary. 


\subsection{Product of kernels}

The following proposition extends Schur lemma about products of reproducing kernels to the vector valued case.

Proposition 9. Let $K$ be a $\mathcal{Y}$-kernel and $\kappa$ a scalar kernel. Define

$$
(\kappa K)(x, t)=\kappa(x, t) K(x, t) \quad x, t \in X,
$$

then $\kappa K$ is a $\mathcal{Y}$-reproducing kernel and $\mathcal{H}_{\kappa K}$ is embedded into $\mathcal{H}_{\kappa} \otimes \mathcal{H}_{K}$ by means of the feature operator

$$
W(\varphi \otimes f)(x)=\varphi(x) f(x) \quad \varphi \in \mathcal{H}_{\kappa}, f \in \mathcal{H}_{K} .
$$

If both $\kappa$ and $K$ are Mercer kernels, so is $\kappa K$, whereas if

$$
\sup _{x \in X}\{\kappa(x, x),\|K(x, x)\|\}<\infty \text { and }\left\{\begin{array}{l}
\kappa_{x} \in \mathcal{C}_{0}(X) \text { and } K_{x} v \in \mathcal{C}(X ; \mathcal{Y}) \\
\text { or } \\
\kappa_{x} \in \mathcal{C}(X) \text { and } K_{x} v \in \mathcal{C}_{0}(X ; \mathcal{Y})
\end{array}\right.
$$

then $K$ is a $\mathcal{C}_{0}$ kernel.

Proof. Let $\mathcal{H}=\mathcal{H}_{\kappa} \otimes \mathcal{H}_{K}$. Since $\kappa$ is a scalar kernel, $\kappa_{x} \in \mathcal{H}_{\kappa}$. Define $\gamma_{x}: \mathcal{Y} \rightarrow \mathcal{H}$ by means of $\gamma_{x} y=\kappa_{x} \otimes K_{x} y$, then $\gamma_{x}^{*}(\varphi \otimes f)=\varphi(x) f(x)$. First claim is a consequence of Proposition 1 .

If both $\kappa$ and $K$ are Mercer kernels, clearly $\kappa K$ is Mercer.

To prove that if (13) hold then $K$ is $\mathcal{C}_{0}$, we apply Corollary 1 with $\mathcal{S}=$ $\left\{\varphi \otimes f \mid \varphi \in \mathcal{H}_{\kappa}, f \in \mathcal{H}_{K}\right\}$, and observe that

$$
\left\|\gamma_{x}\right\| \leq\left\|\kappa_{x}\right\|_{\kappa}\left\|K_{x}\right\| \leq C
$$

by assumption.

Based on the above results, we characterize the RKHS whose kernel is given in [5].

Example 5. Let $\kappa$ be a scalar reproducing kernel and $B$ a positive bounded operator on $\mathcal{Y}$. Define $K: X \times X \rightarrow \mathcal{L}(\mathcal{Y})$ as

$$
K(x, t)=\kappa(x, t) B \quad x, t \in X
$$

(i) The map $K$ is a $\mathcal{Y}$-reproducing kernel and $\mathcal{H}_{K}$ is unitarily equivalent to $\mathcal{H}_{\kappa} \otimes \operatorname{ker} B^{\perp}$ by means of the unitary operator

$$
W(\varphi \otimes y)(x)=\varphi(x) B^{\frac{1}{2}} y .
$$


(ii) If $\kappa$ is Mercer $\left[\right.$ resp. $\left.\mathcal{C}_{0}\right]$, then $K$ is Mercer $\left[\right.$ resp. $\left.\mathcal{C}_{0}\right]$, too.

(iii) If there is an orthonormal basis $\left(y_{i}\right)_{i \in I}$ of $\operatorname{ker} B^{\perp}$ such that $B y_{i}=\sigma_{i} y_{i}$ (so that $\sigma_{i}>0$ for all $i \in I$ ), then $\mathcal{H}_{K}$ is unitarily equivalent to $\oplus_{i \in I} \mathcal{H}_{\kappa}$ by means of the unitary operator

$$
\widetilde{W}\left(\oplus_{i \in I} \varphi_{i}\right)(x)=\sum_{i \in I} \sqrt{\sigma_{i}} \varphi_{i}(x) y_{i}
$$

where the series converges in norm.

Proof. First two items are a consequence of Proposition 9 and Example 2. We prove item (iii) in two steps. Apply first Proposition 6 with $w: \mathcal{Y} \rightarrow \ell_{2}$, $(w y)_{i}=\left\langle y, y_{i}\right\rangle$, so that $\mathcal{H}_{K_{w}}$ is embedded in $\mathcal{H}_{K}$, by means of the feature operator $W_{w}(f)=w \circ f$ for all $f \in \mathcal{H}_{K}$. The corresponding $\ell_{2}$-kernel is $K_{w}(x, t)=\kappa(x, t) w B w^{*}$. By definition of $w$, the kernel $K_{w}$ is diagonal with respect to $\left(e_{i}\right)_{i \in I}$, the canonical basis of $\ell_{2}$, namely

$$
K_{w}(x, t)=\sum_{i \in I} \sigma_{i} \kappa(x, t) e_{i} \otimes \overline{e_{i}}=: \sum_{i \in I} K^{i}(x, t),
$$

where the series converges in the strong operator topology.

Now observe that, for each $i \in I, \operatorname{ker}\left(\sigma_{i} e_{i} \otimes \overline{e_{i}}\right)^{\perp}=\mathbb{C} e_{i}$, so that for item (i) of this example, the space $\mathcal{H}_{K^{i}}$ is unitarily equivalent to $\mathcal{H}_{\kappa} \otimes \mathbb{C} e_{i} \simeq \mathcal{H}_{\kappa}$, through the feature operator

$$
W^{i}: \mathcal{H}_{\kappa} \rightarrow \mathcal{H}_{K^{i}}, \quad W^{i}(\varphi)(x)=\varphi(x) \sqrt{\sigma_{i}} e_{i}
$$

Applying Proposition 5 to the family $\left(K^{i}\right)_{i \in I}$, we obtain a unitary operator

$$
W: \bigoplus_{i \in I} \mathcal{H}_{\kappa} \longrightarrow \mathcal{H}_{K_{w}}, \quad W\left(\oplus_{i} \varphi_{i}\right)(x)=\sum_{i} \varphi_{i}(x) \sqrt{\sigma_{i}} e_{i}
$$

(the operator $W$ is unitary since $\sigma_{i}>0$ for all $i \in I$, so that $W$ is injective). Equation (14) is finally obtained letting $\widetilde{W}=W_{w}^{*} W$.

If in Example 5, $\mathcal{Y}$ is a RKHS of scalar functions over some set $X^{\prime}$, then there is a particular choice for the operator B, suggested by Example 1 .

Example 6. Let $X$ and $X^{\prime}$ be two locally compact second countable topological spaces. Let $\kappa: X \times X \rightarrow \mathbb{C}$ and $\kappa^{\prime}: X^{\prime} \times X^{\prime} \rightarrow \mathbb{C}$ be two scalar reproducing kernels on $X$ and $X^{\prime}$, respectively. 
(i) If $I^{\prime}$ denotes the identity operator on $\mathcal{H}_{\kappa^{\prime}}$, define

$$
K: X \times X \rightarrow \mathcal{L}\left(\mathcal{H}_{\kappa}\right) \quad K(x, t)=\kappa(x, t) I^{\prime},
$$

then $K$ is a $\mathcal{H}_{\kappa^{\prime}}$-reproducing kernel on $X$ and the corresponding $R K H S$ $\mathcal{H}_{K}$ is unitarily equivalent to $\mathcal{H}_{\kappa} \otimes \mathcal{H}_{\kappa^{\prime}}$ by means of the feature operator

$$
W: \mathcal{H}_{\kappa} \otimes \mathcal{H}_{\kappa^{\prime}} \longrightarrow \mathcal{H}_{K}, \quad W\left(\varphi_{1} \otimes \varphi_{2}\right)(x)=\varphi_{1}(x) \varphi_{2} .
$$

(ii) Define $\kappa \times \kappa^{\prime}:\left(X \times X^{\prime}\right) \times\left(X \times X^{\prime}\right) \rightarrow \mathbb{C}$ as

$$
\left(\kappa \times \kappa^{\prime}\right)\left(x, x^{\prime} ; t, t^{\prime}\right)=\kappa(x, t) \kappa^{\prime}\left(x^{\prime}, t^{\prime}\right),
$$

then $\kappa \times \kappa^{\prime}$ is a scalar kernel on $X \times X^{\prime}$ and $\mathcal{H}_{\kappa \times \kappa^{\prime}}$ is unitarily equivalent to $\mathcal{H}_{K}$ by means of the feature operator

$$
\widetilde{W}(f)\left(x, x^{\prime}\right)=[f(x)]\left(x^{\prime}\right)=\left\langle f(x), \kappa_{x^{\prime}}^{\prime}\right\rangle_{\kappa^{\prime}} \quad f \in \mathcal{H}_{K} .
$$

Proof. The first part follows from Example 5 with $\mathcal{Y}=\mathcal{H}_{\kappa^{\prime}}$ and $B=I^{\prime}$, which is injective. The second part is a consequence of Proposition 1 applied to

$$
\gamma: X \times X^{\prime} \longrightarrow \mathcal{L}\left(\mathbb{C} ; \mathcal{H}_{K}\right) \simeq \mathcal{H}_{K}, \quad\left(x, x^{\prime}\right) \longmapsto W\left(\kappa_{x} \otimes \kappa_{x^{\prime}}^{\prime}\right)
$$

taking into account the injectivity of $W$ and the equalities

$$
\begin{aligned}
\left\langle W\left(\varphi_{1} \otimes \varphi_{2}\right), \gamma_{\left(x, x^{\prime}\right)}\right\rangle_{K} & =\left\langle\varphi_{1} \otimes \varphi_{2}, \kappa_{x} \otimes \kappa_{x^{\prime}}^{\prime}\right\rangle=\varphi_{1}(x)\left\langle\varphi_{2}, \kappa_{x^{\prime}}^{\prime}\right\rangle_{\kappa^{\prime}} \\
& =\left\langle W\left(\varphi_{1} \otimes \varphi_{2}\right)(x), \kappa_{x^{\prime}}^{\prime}\right\rangle_{\kappa^{\prime}}=\widetilde{W}\left(W\left(\varphi_{1} \otimes \varphi_{2}\right)\right)\left(x, x^{\prime}\right) .
\end{aligned}
$$

By using Proposition 4 on the space $X^{\prime}$, the above example can be realized in an alternative way.

Example 7. Let $X$ and $X^{\prime}$ be two locally compact second countable topological spaces. Let $\kappa: X \times X \rightarrow \mathbb{C}$ and $\kappa^{\prime}: X^{\prime} \times X^{\prime} \rightarrow \mathbb{C}$ be two scalar $\mathcal{C}_{0}$-reproducing kernels on $X$ and $X^{\prime}$, respectively. Let $\mu^{\prime}$ be a probability measure on $X^{\prime}$ with supp $\mu^{\prime}=X^{\prime}$ and $L_{\mu^{\prime}}$ be the integral operator on $L^{2}\left(X^{\prime}, \mu^{\prime}\right)$ with kernel $\kappa^{\prime}$. Define

$$
\widehat{K}: X \times X \rightarrow \mathcal{L}\left(L^{2}\left(X^{\prime}, \mu^{\prime}\right)\right) \quad \widehat{K}(x, t)=\kappa(x, t) L_{\mu^{\prime}},
$$

then the kernel $\widehat{K}$ is a $L^{2}\left(X^{\prime}, \mu^{\prime}\right)$-reproducing kernel and the space $\mathcal{H}_{\widehat{K}}$ is unitarily equivalent to $\mathcal{H}_{\kappa} \otimes \mathcal{H}_{\kappa^{\prime}}$ by means of

$$
\widehat{W}(f \otimes g)(x)=f(x) i_{\mu^{\prime}}(g) \quad f \in \mathcal{H}_{\kappa}, g \in \mathcal{H}_{\kappa^{\prime}},
$$

where $i_{\mu^{\prime}}$ is the inclusion of $\mathcal{H}_{\kappa^{\prime}}$ in $L^{2}\left(X^{\prime}, \mu\right)$. In particular, $\widehat{K}$ is a $\mathcal{C}_{0}$-kernel. 
Proof. Apply Proposition [6 with $K=\kappa I^{\prime}$, as in the previous example, and $w=i_{\mu^{\prime}}$, which is injective. Clearly $K_{w}=\widehat{K}$, so that $\mathcal{H}_{\widehat{K}}$ is unitarily equivalent to $\mathcal{H}_{\kappa I^{\prime}}$. The thesis follows immediately from Example 6 .

The above example shows that $\mathcal{H}_{K}$ and $\mathcal{H}_{\widehat{K}}$ are the same RKHS, where the elements of $\mathcal{H}_{K}$ are regarded as functions from $X$ into $\mathcal{H}_{\kappa^{\prime}}$, whereas the elements of $\mathcal{H}_{\widehat{K}}$ are regarded as functions from $X$ into $L^{2}\left(X^{\prime}, \mu^{\prime}\right)$.

\subsection{Application to learning theory}

We end this section considering an application of some of the above examples to vector valued regression problems. In learning theory, a popular algorithm is the minimization on a RKHS $\mathcal{H}_{K}$ of the empirical error with a penalty term proportional to the square of the norm [13], namely

$$
f^{\star}=\underset{f \in \mathcal{H}_{K}}{\operatorname{argmin}}\left(\frac{1}{n} \sum_{\ell=1}^{n}\left\|y^{\ell}-f\left(x^{\ell}\right)\right\|_{\mathcal{Y}}^{2}+\lambda\|f\|_{K}^{2}\right) .
$$

Here $\left\{\left(x^{1}, y^{1}\right), \ldots,\left(x^{n}, y^{n}\right)\right\}$ is the training set of $n$ input-output pairs $\left(x^{\ell}, y^{\ell}\right) \in$ $X \times Y$ and $\lambda>0$ is the regularization parameter. If the reproducing kernel $K$ is as in Example 5, then it can be checked that

$$
f^{\star}(x)=\sum_{i \in I} \varphi_{i}^{\star}(x) y_{i}
$$

where each $\varphi_{i}^{\star}$ is given by

$$
\varphi_{i}^{\star}=\underset{\varphi \in \mathcal{H}_{\kappa}}{\operatorname{argmin}}\left(\frac{1}{n} \sum_{\ell=1}^{n}\left|y_{i}^{\ell}-\varphi\left(x^{\ell}\right)\right|^{2}+\frac{\lambda}{\sigma_{i}}\|\varphi\|_{\kappa}^{2}\right),
$$

and $y_{i}^{\ell}=\left\langle y^{\ell}, y_{i}\right\rangle$.

In many applications $\mathcal{Y}=\mathbb{C}^{m}$ so that $B$ is a $m \times m$ positive semi-definite matrix. The above observation reduces the problem of computing the minimizer of (15) to $|I|$ scalar problems, where the cardinality $|I|$ is the rank of the matrix $B$.

With the choice of $K$ as in Proposition 8 , let $f^{\star}$ be the minimizer given by (15), where the $n$-examples in the training set are the pairs $\left(t^{\ell}, y^{\ell}\right) \in$ $T \times \mathbb{R}^{m}$. By using the fact that $W$ is a partial surjective isometry, one can check that

$$
f^{\star}(t)=\left(\varphi^{\star}\left(\Psi_{i}(t)\right), \ldots, \varphi^{\star}\left(\Psi_{m}(t)\right)\right.
$$


where $\varphi^{\star}$ is given by

$$
\varphi^{\star}=\underset{\varphi \in \mathcal{H}_{\kappa}}{\operatorname{argmin}}\left(\frac{1}{n} \sum_{\ell=1}^{n} \sum_{i=1}^{m}\left|y_{i}^{\ell}-\varphi\left(x_{i}^{\ell}\right)\right|^{2}+\lambda\|\varphi\|_{\kappa}^{2}\right),
$$

where $y_{i}^{\ell} \in \mathbb{R}$ are the components of the output $y^{\ell} \in \mathbb{R}^{m}$ and $x_{i}^{\ell}=\Psi_{i}\left(t^{\ell}\right) \in X$. With this choice the problem (15) is reduced to a minimization problem on the scalar RKHS $\mathcal{H}_{\kappa}$.

\section{Universal kernels: main results}

In this section we address the problem of defining and characterizing the universality of a kernel $K$. As pointed out in the introduction, in learning theory a necessary condition in order to have universally consistent algorithms is the assumption that the reproducing kernel Hilbert space $\mathcal{H}_{K}$ is dense in $L^{2}(X, \mu ; \mathcal{Y})$ for any probability measure $\mu$. From this point of view next definition is very natural.

Definition 2. Let $K: X \times X \rightarrow \mathcal{L}(\mathcal{Y})$ be a reproducing kernel.

(i) $A \mathcal{C}_{0}$-kernel $K$ is called universal if $\mathcal{H}_{K}$ is dense in $L^{2}(X, \mu ; \mathcal{Y})$ for each probability measure $\mu$.

(ii) A Mercer kernel $K$ is called compact-universal if $\mathcal{H}_{K}$ is dense in $L^{2}(X, \mu ; \mathcal{Y})$ for each probability measure $\mu$ with compact support.

We briefly comment on the above definitions. In item (i) the assumption that the kernel is $\mathcal{C}_{0}$ ensures both that $\mathcal{H}_{K}$ is a subspace of $L^{2}(X, \mu ; \mathcal{Y})$ and that universality is equivalent to the density of $\mathcal{H}_{K}$ is $\mathcal{C}_{0}(X ; \mathcal{Y})$ (see Theorem (1). In item (ii), since $\mu$ has compact support, it is enough to assume that $K$ is a Mercer kernel in order to have $\mathcal{H}_{K} \subset L^{2}(X, \mu ; \mathcal{Y})$. This last property turns out to be equivalent to the definition of universality given in [5].

Clearly a universal kernel is also compact-universal. Conversely, a $\mathcal{C}_{0}$-kernel can be compact-universal but not universal, as shown by Examples 8 and 11 .

Notice that in Definition 2 if we replace $L^{2}(X, \mu ; \mathcal{Y})$ with $L^{p}(X, \mu ; \mathcal{Y})$ for an arbitrary $1 \leq p<\infty$, we have in principle a different notion of universality. Nevertheless Theorem 1 clarifies that there is no difference. We state the results for $p=2$, since it is the natural choice in learning theory.

The following corollary shows that universality is preserved by restriction to a subset. 
Corollary 3. Let $X_{0}$ be a subset of $X$.

(i) If $X_{0}$ is closed and $K$ is universal, then $K_{X_{0}}$ is universal.

(ii) If $X_{0}$ is locally closed and $K$ is compact-universal, then $K_{X_{0}}$ is compactuniversal.

Proof. We only prove (i). Since $X_{0}$ is closed, Corollary 2 implies that $K_{X_{0}}$ is a $\mathcal{C}_{0}$-kernel, and a function $f$ belongs to $\mathcal{H}_{K_{X_{0}}}$ if and only if there exists $g \in \mathcal{H}_{K}$ such that $f=g_{\left.\right|_{X_{0}}}$. Given a probability measure $\mu$ on $X_{0}$, let $\nu$ be the probability measure on $X, \nu(E)=\mu\left(E \cap X_{0}\right)$ for any Borel subset $E$ of $X$. By universality of $K, \mathcal{H}_{K}$ is dense in $L^{2}(X, \nu, \mathcal{Y}) \simeq L^{2}\left(X_{0}, \mu, \mathcal{Y}\right)$, where the equivalence is given by the restriction from $X$ to $X_{0}$, so that $\mathcal{H}_{K_{X_{0}}}$ is dense in $L^{2}\left(X_{0}, \mu, \mathcal{Y}\right)$.

The converse is clearly not true. Notice that the compact-universal kernels are precisely the Mercer kernels such that $K_{X_{0}}$ is universal for any compact subset $X_{0}$ of $X$.

In the next subsections we discuss separately the two notions of universality and then we make a comparison between them.

\subsection{Universality and $\mathcal{C}_{0}$-kernels}

In this section we characterize the universal $\mathcal{C}_{0}$-kernels. First result shows that the density of $\mathcal{H}_{K}$ in $L^{2}(X, \mu ; \mathcal{Y})$ for any probability measure $\mu$ is equivalent to the density in $\mathcal{C}_{0}(X ; \mathcal{Y})$ and that one can replace $L^{2}(X, \mu ; \mathcal{Y})$ with $L^{p}(X, \mu ; \mathcal{Y}), 1 \leq p<\infty$.

Theorem 1. Suppose $K$ is a $\mathcal{C}_{0}$-kernel. The following facts are equivalent.

(a) The kernel $K$ is universal.

(b) The space $\mathcal{H}_{K}$ is dense in $\mathcal{C}_{0}(X ; \mathcal{Y})$.

(c) There is $1 \leq p<\infty$ such that $\mathcal{H}_{K}$ is dense in $L^{p}(X, \mu ; \mathcal{Y})$ for all probability measures $\mu$ on $X$.

Proof. Clearly (a) implies (c). Since $X$ is locally compact and second countable, $\mathcal{C}_{0}(X ; \mathcal{Y})$ is dense in $L^{2}(X, \mu ; \mathcal{Y})$ where the inclusion is continuous, so that (b) implies (a).

We show that (c) implies (b). Suppose hence that $\mathcal{H}_{K}$ is not dense in $\mathcal{C}_{0}(X ; \mathcal{Y})$. Then, there exists $T \in \mathcal{C}_{0}(X ; \mathcal{Y})^{*}, T \neq 0$ such that $T(f)=0$ for all $f \in \mathcal{H}_{K}$. By Theorem 7 , there is a probability measure $\mu$ on $X$ and a function $h \in L^{\infty}(X, \mu ; \mathcal{Y})$ such that $T(f)=\int_{X}\langle f(x), h(x)\rangle \mathrm{d} \mu(x)$. Since 
$T \neq 0$, then $h \neq 0$.

Since $\mu$ is a probability measure, $h$ is a non-null element in $L^{p /(p-1)}(X, \mu ; \mathcal{Y})=$ $L^{p}(X, \mu ; \mathcal{Y})^{*}($ where we set $1 / 0=\infty)$ such that

$$
\int_{X}\langle f(x), h(x)\rangle \mathrm{d} \mu(x)=0 \quad \forall f \in \mathcal{H}_{K} .
$$

It follows that $\mathcal{H}_{K}$ is not dense in $L^{p}(X, \mu ; \mathcal{Y})$.

As a consequence of the previous theorem, we have the following nice corollary.

Corollary 4. Let $K$ be a $\mathcal{C}_{0}$ - kernel. Given $1 \leq p \leq q<\infty$, the space $\mathcal{H}_{K}$ is dense in $L^{p}(X, \mu ; \mathcal{Y})$ for all probability measures $\mu$ if and only if it is dense in $L^{q}(X, \mu ; \mathcal{Y})$ for all probability measures $\mu$.

The previous result is not trivial. Clearly, if $q \geq p$, the space $L^{q}(X, \mu ; \mathcal{Y})$ is always a dense subspace of $L^{p}(X, \mu ; \mathcal{Y})$ and the inclusion is continuous. Hence, if a RKHS $\mathcal{H}_{K}$ is dense in $L^{q}(X, \mu ; \mathcal{Y})$, then $\mathcal{H}_{K}$ is always dense in $L^{p}(X, \mu ; \mathcal{Y})$. However, in general $L^{p}(X, \mu ; \mathcal{Y})$ is not contained in $L^{q}(X, \mu ; \mathcal{Y})$, so that, if $\mathcal{H}_{K}$ is dense $L^{p}(X, \mu ; \mathcal{Y})$, the density of $\mathcal{H}_{K}$ in $L^{q}(X, \mu ; \mathcal{Y})$ has to be proved. Corollary 4 shows this result under the assumption that $K$ is $\mathcal{C}_{0}$.

Now, we give a characterisation of universality of $K$ in terms of the injectivity property of the integral operators $L_{\mu}$, for $\mu$ varying over the probability measures on $X$.

Theorem 2. Suppose $K$ is a $\mathcal{C}_{0}$-kernel. Then the following facts are equivalent.

(a) The kernel $K$ is universal.

(b) The operator $i_{\mu}^{*}: L^{2}(X, \mu ; \mathcal{Y}) \rightarrow \mathcal{H}_{K}$ is an injective operator for all probability measures $\mu$ on $X$.

(c) The integral operator $L_{\mu}: L^{2}(X, \mu ; \mathcal{Y}) \rightarrow L^{2}(X, \mu ; \mathcal{Y})$ is injective for all probability measures $\mu$ on $X$.

The proof is an immediate consequence of Theorem 1 and the next proposition.

Proposition 10. Let $K$ be a Mercer kernel and $\mu$ a fixed probability measure on $X$ such that $K$ is bounded on the support of $\mu$. The following facts are equivalent.

(a) The space $\mathcal{H}_{K}$ is dense in $L^{2}(X, \mu ; \mathcal{Y})$. 
(b) The operator $i_{\mu}^{*}$ is injective.

(c) The integral operator $L_{\mu}$ is injective.

Proof. The space $\mathcal{H}_{K}$ is dense in $L^{2}(X, \mu ; \mathcal{Y})$ if and only if the range of $i_{\mu}$ is dense in $L^{2}(X, \mu ; \mathcal{Y})$. This last condition is equivalent to the injectivity of $i_{\mu}^{*}$, that is, (a) is equivalent to (b). Since $L_{\mu}=i_{\mu} i_{\mu}^{*}$ and $\operatorname{ker} L_{\mu}=\operatorname{ker} i_{\mu}^{*}$, then (b) and (c) are equivalent.

\subsection{Compact-universality}

In this section, we characterize compact-universality of Mercer kernels and we show that compact-universality is precisely what is called universality in [5].

Next theorem characterizes compact-universality.

Theorem 3. Suppose $K$ is a Mercer kernel. The following facts are equivalent.

(a) The kernel $K$ is compact-universal.

(b) The space $\mathcal{H}_{K}$ is dense in $\mathcal{C}(X ; \mathcal{Y})$ endowed with compact-open topology.

(c) There is $1 \leq p<\infty$ such that $\mathcal{H}_{K}$ is dense in $L^{p}(X, \mu ; \mathcal{Y})$ for all compactly supported probability measures.

Proof. Clearly (a) implies (c). We prove that (b) implies (a). Indeed, fixed a probability measure $\mu$ with compact support $Z$, the fact that $\mathcal{H}_{K}$ is dense in $\mathcal{C}(X ; \mathcal{Y})$ implies that $\left.\mathcal{H}_{K}\right|_{Z}:=\left\{f_{\left.\right|_{Z}} \mid f \in \mathcal{H}_{K}\right\}$ is dense in $\mathcal{C}(Z ; \mathcal{Y})$, but $\mathcal{C}(Z ; \mathcal{Y})$ is clearly dense in $L^{2}(Z, \mu ; \mathcal{Y}) \simeq L^{2}(X, \mu ; \mathcal{Y})$ with continuous injection. Hence $\mathcal{H}_{K}$ is dense in $L^{2}(X, \mu ; \mathcal{Y})$. It only remains to prove that (c) implies (b). For this, it is enough to prove that $\left.\mathcal{H}_{K}\right|_{Z}$ is dense in $\mathcal{C}(Z ; \mathcal{Y})$ with the uniform norm, for all compact subset $Z$ of $X$. But this is a simple consequence of Theorem 1 since $\left.\mathcal{H}_{K}\right|_{Z}$ is clearly dense in $L^{p}(Z, \mu ; \mathcal{Y})$ for all probability measure $\mu$ on $Z$, and $\mathcal{C}(Z ; \mathcal{Y})=\mathcal{C}_{0}(Z ; \mathcal{Y})$.

The analog of theorem 2 also holds.

Theorem 4. Suppose $K$ is a Mercer kernel. Then the following facts are equivalent.

(a) The kernel $K$ is compact-universal.

(b) The operator $i_{\mu}^{*}: \mathcal{H}_{K} \rightarrow L^{2}(X, \mu ; \mathcal{Y})$ is an injective operator for all compactly supported probability measures $\mu$ on $X$. 
(c) The integral operator $L_{\mu}: L^{2}(X, \mu ; \mathcal{Y}) \rightarrow L^{2}(X, \mu ; \mathcal{Y})$ is injective for all probability measures $\mu$ on $X$ with compact support.

The proof is a simple consequence of Proposition 10.

Clearly universality of a $\mathcal{C}_{0}$-kernel $K$ implies compact-universality. The converse is not true as shown by the following example, see also Example 11. The reason of this phenomenon is the fact that $\mathcal{C}_{0}(X ; \mathcal{Y})$ endowed with the compact-open topology is not continuously embedded in $L^{p}(X, \mu ; \mathcal{Y})$.

Example 8. Let $X=\mathbb{Z}_{+}$, and let $\ell^{2}$ be the Hilbert space of square summable sequences. Then, $\ell^{2}$ is a RKHS of scalar functions on $X$ with reproducing kernel $K(i, j)=\delta_{i, j}$, where $\delta_{i, j}$ is the Kronecker delta. We fix the following sequence $\left\{f_{k}\right\}_{k \in \mathbb{Z}_{+}}$in $\ell^{2}$

$$
f_{k}(j)=\delta_{j, k}+e \delta_{j, k+1},
$$

and we let

$$
\mathcal{H}_{\tilde{K}}=\ell^{2}-\operatorname{cl} \operatorname{span}\left\{f_{k} \mid k \in \mathbb{Z}_{+}\right\}
$$

$\left(\ell^{2}-\mathrm{cl}\right.$ denotes the closure in $\left.\ell^{2}\right) . \mathcal{H}_{\tilde{K}}$ is also a RKHS of scalar functions on $X$, whose reproducing kernel we denote by $\tilde{K}$. Since $\ell^{2} \subset c_{0}(=$ the sequences going to 0 at infinity), $\tilde{K}$ is a $\mathcal{C}_{0}$-reproducing kernel.

For all $n \in \mathbb{Z}_{+}$, let $Z_{n}=\{1,2 \ldots n\} . Z_{n}$ is compact in $X$, and every compact set $Z \subset X$ is contained in some $Z_{n}$. Clearly,

$$
\mathcal{C}\left(Z_{n}\right)=\operatorname{span}\left\{\left(f_{k}\right)_{\left.\right|_{n}} \mid k \leq n\right\}
$$

hence $\mathcal{H}_{\tilde{K}}$ is dense in $\mathcal{C}(X)$ with the topology of uniform convergence on compact subsets.

Let $\mu$ be the probability measure on $X$ such that $\mu(\{j\})=(e-1) e^{-j}$. We claim that $\mathcal{H}_{\tilde{K}}$ is not dense in $L^{2}(X, \mu)$. In fact, let $f \in L^{2}(X, \mu)$ be the function $f(j)=(-1)^{j}$. We have $\left\langle f_{k}, f\right\rangle_{L^{2}(X, \mu)}=0$ for all $k$. By (16) and continuity of the inclusion $\ell^{2} \hookrightarrow L^{2}(X, \mu)$, we see that $f$ is in the orthogonal complement of $\mathcal{H}_{\tilde{K}}$ in $L^{2}(X, \mu)$. The claim then follows.

A universal kernel is strictly positive definite, but the converse in general fails, as shown by the following corollary and example.

Corollary 5. Suppose $K$ is a compact-universal kernel. Then $K$ is strictly positive definite, i.e. for all finite subsets $\left\{x_{1}, x_{2} \ldots x_{N}\right\}$ of $X$ such that $x_{i} \neq$ $x_{j}$ if $i \neq j$, the condition

$$
\sum_{i, j=1}^{N}\left\langle K\left(x_{i}, x_{j}\right) y_{j}, y_{i}\right\rangle=0 \quad\left(y_{i} \in \mathcal{Y}, i=1 \ldots N\right)
$$

implies $y_{i}=0$ for all $i=1, \ldots, N$. 
Proof. Assume $\sum_{i, j=1}^{N}\left\langle K\left(x_{i}, x_{j}\right) y_{j}, y_{i}\right\rangle=0$ for some finite subset $\left\{x_{1}, x_{2} \ldots x_{N}\right\} \in$ $X, x_{i} \neq x_{j}$ if $i \neq j$, and $\left\{y_{1}, y_{2} \ldots x_{N}\right\}$ in $\mathcal{Y}$. Taking

$$
\mu=\frac{1}{N} \sum_{i=1}^{N} \delta_{x_{i}} \quad \text { and } \quad \varphi=\sum_{i=1}^{N} y_{i} \delta_{x_{i}},
$$

we obtain a probability measure $\mu$ on $X$ with compact support and a function $\varphi \in L^{2}(X, \mu ; \mathcal{Y})$ such that

$$
\begin{aligned}
0 & =\sum_{i, j=1}^{N}\left\langle K\left(x_{i}, x_{j}\right) y_{j}, y_{i}\right\rangle=N^{2} \int_{X \times X}\langle K(x, y) \varphi(y), \varphi(x)\rangle d \mu(y) d \mu(x) \\
& =N^{2} \int_{X}\left\langle\left(L_{\mu} \varphi\right)(x), \varphi(x)\right\rangle d \mu(x)=N^{2}\left\langle L_{\mu} \varphi, \varphi\right\rangle_{2} .
\end{aligned}
$$

Since $L_{\mu}$ is positive and injective by Theorem 4, we have $\varphi\left(x_{i}\right)=0$ for all $i=1, \ldots, N$. Since $x_{i} \neq x_{j}$ if $i \neq j$, then $y_{i}=0$ for all $i=1, \ldots, N$.

The converse of the above corollary fails to be true, as shown by the following example.

Example 9. Let $K: \mathbb{R} \times \mathbb{R} \rightarrow \mathbb{C}$ be the kernel

$$
K(x, t)=\int_{-1}^{1} e^{2 \pi i(x-t) p} \mathrm{~d} p=\frac{\sin 2 \pi(x-t)}{\pi(x-t)} .
$$

The map $K$ is a scalar $\mathcal{C}_{0}$-kernel, which is strictly positive definite, but not universal.

Proof. We show that it is strictly positive definite. Indeed, let $x_{1}, \ldots x_{N} \in X$ such that $x_{i} \neq x_{j}$ if $i \neq j, c_{1}, \ldots, c_{N} \in \mathbb{C}$ and suppose

$$
0=\sum_{i, j=1}^{N} c_{i} \overline{c_{j}} K\left(x_{i}, x_{j}\right)=\int_{-1}^{1}\left|\sum_{i=1}^{N} c_{i} e^{2 \pi i x_{i} p}\right|^{2} \mathrm{~d} p
$$

Since $p \mapsto\left|\sum_{i} c_{i} e^{2 \pi i x_{i} p}\right|^{2}$ is continuous, it follows that $\left|\sum_{i} c_{i} e^{2 \pi i x_{i} p}\right|^{2}=0$ for all $p \in[-1,1]$. Observing that the functions $f_{j}(t)=e^{2 \pi i x_{j} t}$ are linearly independent on $[-1,1]$ since $x_{i} \neq x_{j}$, it follows that $c_{j}=0$ for all $j$. Clearly $K$ is a $\mathcal{C}_{0}$-kernel, but it is not universal (see Example [11).

In the next remark we show that compact-universality is exactly what is called universality in [5]. 
Remark 1. In [5], a Mercer kernel $K$ is said to be universal if, for each compact set $Z \subseteq X$

$$
\mathcal{C}(Z ; \mathcal{Y})=\|\cdot\|_{Z}-\mathrm{cl} \operatorname{span}\left\{K(\cdot, x) v_{\mid Z} \mid x \in Z, v \in \mathcal{Y}\right\}
$$

where $\|\cdot\|_{Z}-\mathrm{cl}$ denotes the closure in $\mathcal{C}(Z ; \mathcal{Y})$ with the uniform norm topology. This is equivalent to require that $\mathcal{H}_{K}$ is dense $\mathcal{C}(X ; \mathcal{Y})$ with the compactopen topology, that is, by Theorem 1 that $K$ is compact-universal. Indeed, by definition of the compact-open topology, $\mathcal{H}_{K}$ is dense in $\mathcal{C}(X ; \mathcal{Y})$ if and only if

$$
\mathcal{C}(Z ; \mathcal{Y})=\|\cdot\|_{Z}-\left.\operatorname{cl} \mathcal{H}_{K}\right|_{Z}
$$

for all compact $Z \subseteq X$.

Clearly (17) implies (18). Suppose on the other hand that (18) holds true. Denote with $\widetilde{K}$ the restriction of $K$ to $Z \times Z$. Since convergence in $\mathcal{H}_{\widetilde{K}}$ implies uniform convergence we have

$$
\|\cdot\|_{Z}-\operatorname{cl} \operatorname{span}\left\{K(\cdot, x) v_{\left.\right|_{Z}} \mid x \in Z, v \in \mathcal{Y}\right\} \supseteq \mathcal{H}_{\widetilde{K}}
$$

On the other hand, $\mathcal{H}_{\widetilde{K}}=\left.\mathcal{H}_{K}\right|_{Z}$ as a linear space of functions (see Corollary 21). Hence (18) implies (17).

\section{Translation invariant kernels and univer- sality}

In this section we assume that $X$ is a locally compact second countable topological group with identity $e$ and we study the reproducing kernels that are translation invariant, namely

$$
K(z x, z t)=K(x, t) \quad \text { for all } x, t, z \in X .
$$

In particular we characterize all the translation invariant kernels in terms of a unitary representation of $X$ acting on an arbitrary Hilbert space $\mathcal{H}$ and an operator $A: \mathcal{H} \rightarrow \mathcal{Y}$. If $X$ is an abelian group, we give a more explicit characterization in Theorem 5 and Theorem 13 provides a sufficient condition ensuring that the corresponding reproducing kernel Hilbert space is universal. This condition is also necessary if $X$ is compact or $\mathcal{Y}=\mathbb{C}$. For scalar kernels on $\mathbb{R}^{d}$ our result has been already proved in [22].

For a representation $\pi$ of $X$ on a vector space $V$ we mean a group homomorphism from $X$ to the automorphisms of $V$. In particular, if $V$ is a Hilbert space, $\pi$ is unitary if it takes values in the group of unitary operators 
on $V$. In this framewok the representation is called continuous if $\pi$ is strongly continuous (see [16]).

We denote by $\lambda$ the left regular representation of $X$ acting on $\mathcal{F}(X ; \mathcal{Y})$, namely

$$
\left(\lambda_{x} f\right)(t)=f\left(x^{-1} t\right) \quad t, x \in X, f \in \mathcal{F}(X ; \mathcal{Y}) .
$$

We recall that a function $\Gamma: X \rightarrow \mathcal{L}(\mathcal{Y})$ is of completely positive type if

$$
\sum_{i, j=1}^{N}\left\langle\Gamma\left(x_{j}^{-1} x_{i}\right) y_{j}, y_{i}\right\rangle \geq 0
$$

for all finite sequences $\left\{x_{i}\right\}_{i=1 \ldots N}$ in $X$ and $\left\{y_{i}\right\}_{i=1 \ldots N}$ in $\mathcal{Y}$.

The following facts are easy to prove.

Proposition 11. Let $K: X \times X \rightarrow \mathcal{L}(\mathcal{Y})$ be a reproducing kernel. The following conditions are equivalent.

(a) $K$ is a translation invariant reproducing kernel.

(b) There is a function $K_{e}: X \rightarrow \mathcal{L}(\mathcal{Y})$ of completely positive type such that $K(x, t)=K_{e}\left(t^{-1} x\right)$.

If one the above conditions is satisfied, then the representation $\lambda$ leaves invariant $\mathcal{H}_{K}$, its action on $\mathcal{H}_{K}$ is unitary and

$$
\begin{aligned}
& K(x, t)=K_{e}^{*} \lambda_{x^{-1} t} K_{e} \quad x, t \in X \\
& \|K(x, x)\|=\left\|K_{e}(e)\right\|
\end{aligned}
$$

The notation $K_{e}$ for the function of completely positive type associated with the reproducing kernel $K$ is consistent with the definition given by (11) since

$$
\left(K_{e} y\right)(x)=K_{e}(x) y \quad y \in \mathcal{Y}, x \in X .
$$

Proof of Proposition 11. Assume (a). Given $x, t \in X$, (11) and (19) give

$$
K_{e}\left(t^{-1} x\right)=K\left(t^{-1} x, e\right)=K(x, t) .
$$

Since $K$ is a reproducing kernel, $K_{e}$ is of completely positive type, so that (b) holds true.

Assume (b). Clearly $K$ is a translation invariant reproducing kernel, so that (a) holds true.

Suppose now that $K$ is a translation invariant reproducing kernel. Observe that, given $t \in X$ and $y \in \mathcal{Y}$,

$$
\left(\lambda_{x} K_{t} y\right)(z)=\left(K_{t} y\right)\left(x^{-1} z\right)=K\left(x^{-1} z, t\right) y=K(z, x t) y=\left(K_{x t} y\right)(z) \quad x, z \in X,
$$


that is, $\lambda_{x} K_{t}=K_{x t}$. Moreover

$$
\begin{aligned}
\left\langle\lambda_{x} K_{t_{1}} y_{1}, \lambda_{x} K_{t_{2}} y_{2}\right\rangle_{K} & =\left\langle K_{x t_{1}} y_{1}, K_{x t_{2}} y_{2}\right\rangle_{K}=\left\langle K\left(x t_{2}, x t_{1}\right) y_{1}, y_{2}\right\rangle \\
& =\left\langle K\left(t_{2}, t_{1}\right) y_{1}, y_{2}\right\rangle=\left\langle K_{t_{1}} y_{1}, K_{t_{2}} y_{2}\right\rangle_{K} .
\end{aligned}
$$

This means that $\lambda$ leaves the set $\left\{K_{x} y \mid x \in X, y \in \mathcal{Y}\right\}$ invariant and its action is unitary. First two claims now follow recalling that $\left\{K_{x} y \mid x \in X, y \in \mathcal{Y}\right\}$ is total in $\mathcal{H}_{K}$. To prove (21) observe that

$$
K(x, t)=K_{x}^{*} K_{t}=K_{e}^{*} \lambda_{x}^{*} \lambda_{t} K_{e}=K_{e}^{*} \lambda_{x^{-1} t} K_{e}
$$

for all $x, t \in X$.

Notice that, if $K$ is a translation invariant kernel, (22) implies that the elements of $\mathcal{H}_{K}$ are bounded functions. The following lemma characterizes the translation invariant kernels that are Mercer or $\mathcal{C}_{0}$.

Lemma 1. Let $K_{e}: X \rightarrow \mathcal{Y}$ be a function of completely positive type and let $K$ be the corresponding translation invariant reproducing kernel. The following conditions are equivalent.

(a) The map $K$ is a Mercer kernel.

(b) For all $y \in \mathcal{Y}, K_{e}(\cdot) y \in \mathcal{C}(X ; \mathcal{Y})$.

(c) The representation $\lambda$ is continuous on $\mathcal{H}_{K}$.

Moreover, the map $K$ is a $\mathcal{C}_{0}$-kernel if and only if $K_{e}(\cdot) y \in \mathcal{C}_{0}(X ; \mathcal{Y})$ for all $y \in \mathcal{Y}$.

Proof. The equivalence between (a) and (b) as well as the statement about $\mathcal{C}_{0}$-kernel is a consequence of Proposition 2, observing that $\left(K_{x} y\right)(t)=K_{e}\left(x^{-1} t\right) y$ and (22) holds.

Assume that $K$ is a Mercer kernel. Since $\lambda$ is a unitary representation and the set $\left\{K_{t} y \mid t \in X, y \in \mathcal{Y}\right\}$ is total in $\mathcal{H}_{K}$, it is enough to check that for any $t \in X$ and $y \in \mathcal{Y}$ the function $x \mapsto \lambda_{x} K_{t} y$ is continuous at the identity. Indeed, observe that

$$
\begin{aligned}
\left\|\lambda_{x} K_{t} y-K_{t} y\right\|_{K}^{2} & =\left\|K_{x t} y-K_{t} y\right\|_{K}^{2} \\
& =\langle(K(x t, x t)-K(t, x t)-K(x t, t)+K(t, t)) y, y\rangle, \\
& =\left\langle\left(2 K_{e}(e)-K_{e}\left(t^{-1} x^{-1} t\right)-K_{e}\left(t^{-1} x t\right)\right) y, y\right\rangle
\end{aligned}
$$

which is continuous at the identity by assumption on $K_{e}$. Conversely, if $\lambda$ is continuous, (21) gives that

$$
K_{e}(x) y=K(x, e) y=K_{e}^{*} \lambda_{x^{-1}} K_{e} y,
$$

so that $K_{e}(\cdot) y$ is continuous. 
The following theorem characterizes the translation invariant reproducing kernels.

Proposition 12. Let $\pi$ be a unitary representation of $X$ acting on a separable Hilbert space $\mathcal{H}$ and $A: \mathcal{H} \rightarrow \mathcal{Y}$ a bounded operator. Define

$$
W: \mathcal{H} \rightarrow \mathcal{F}(X ; \mathcal{Y}), \quad(W v)(x)=A \pi_{x^{-1}} v
$$

$W$ is a unitary map from ker $W^{\perp}$ onto the reproducing kernel Hilbert space $\mathcal{H}_{K}$ with translation invariant kernel

$$
K(x, t)=A \pi_{x^{-1} t} A^{*} \quad x, t \in X .
$$

Moreover $W$ intertwines the representations $\pi$ and $\lambda$. Finally $W$ is unitary if and only if the only $\pi$-invariant closed subspace of $\operatorname{ker} A$ is the null space.

Proof. Define $\gamma_{x}: \mathcal{Y} \rightarrow \mathcal{H}$ as $\gamma_{x}=\pi_{x} A^{*}$, so that $(W v)(x)=\gamma_{x}^{*} v=A \pi_{x^{-1}} v$. The claim is now consequence of Proposition 1, up the last statement. The fact that $W$ intertwines $\pi$ with $\lambda$ is trivial. Finally, by Proposition 1, $W$ is unitary if and only if is injective. By definition

$$
\operatorname{ker} W=\left\{v \in \mathcal{H} \mid \pi_{x} v \in \operatorname{ker} A \forall x \in X\right\} \text {. }
$$

Hence ker $W$ is a closed subspace of ker $A$ invariant with respect to $\pi$. Conversely any $\pi$-invariant closed subspace of $\operatorname{ker} A$ is contained in ker $W$.

Proposition 11 and 12 show that any translation invariant kernel is of the form $K(x, t)=A \pi_{x^{-1}{ }_{t}} A^{*}$ for some unitary representation $\pi$ acting on a Hilbert space $\mathcal{H}$ and a bounded operator $A: \mathcal{H} \rightarrow \mathcal{Y}$. In particular, if $\pi$ is a continuous representation, then $K$ is a Mercer kernel and for any Mercer kernel $\pi$ can be assumed to be continuous and $\mathcal{H}$ separable. Moreover, the reproducing kernel Hilbert space $\mathcal{H}_{K}$ is embedded in $\mathcal{H}$ by the feature operator $W$ defined by (23). Observe that if the representation $\pi$ is irreducible or if $A$ is injective, then $W$ is unitary.

If $\mathcal{Y}=\mathbb{C}$, the operator $A$ is of the form $A v=\langle v, w\rangle_{\mathcal{H}}$ for some $w \in \mathcal{H}$, so that $(W v)(x)=\left\langle v, \pi_{x} w\right\rangle_{\mathcal{H}}$. This operator is well know in harmonic analysis as wavelet operator [17].

Remark 2. Notice that any translation invariant kernel $K$ is the sum of translation invariant kernels associated with cyclic representations. Indeed, let $\pi$ be a unitary representation defining $K$ by means of (24). Since any unitary representation is the direct sum of a family of cyclic representations, then $\mathcal{H}=\oplus_{i \in I} \mathcal{H}_{i}$ where each $\mathcal{H}_{i}$ is a closed $\pi$-invariant subspace and the 
action of $\pi$ on $\mathcal{H}_{i}$ is cyclic. Denote by $P_{i}$ the orthogonal projection on $\mathcal{H}_{i}$, then

$$
K(x, t)=\sum_{i \in I} A P_{i} \pi_{x^{-1} t} P_{i} A^{*}=\sum_{i \in I} K^{i}(x, t),
$$

where the series converges in the strong operator topology and the reproducing kernels $K^{i}$ are $K^{i}(x, t)=A_{i} \pi_{x^{-1} t}^{i} A_{i}^{*}$ where $\pi^{i}$ and $A_{i}$ are the restrictions of $\pi$ and $A$ to $\mathcal{H}_{i}$, respectively. Proposition 5 implies that $\mathcal{H}_{K}=\sum_{i \in I} \mathcal{H}_{K^{i}}$. For scalar kernels, we can always assume that $\pi$ is cyclic itself. Indeed, the wavelet operator is $(W v)(x)=\left\langle v, \pi_{x} w\right\rangle_{\mathcal{H}}$ for some $w \in \mathcal{H}$, so that the associated kernel $K$ is determined only by the cyclic subrepresentation of $\pi$ containing $w$.

\subsection{Abelian groups}

In this section, we specialize the previous discussion to the case in which $X$ is an abelian group. With this assumption, we can give a more explicit construction of translation invariant Mercer kernels, which is related to a generalization of Bochner theorem for scalar functions of positive type, [2, 15].

We denote the product in $X$ additively and the identity by 0 , since the main example is $\mathbb{R}^{d}$. We let $\hat{X}$ be the dual group of $X$ and we denote by $\mathrm{d} x$ the Haar measure on $X$.

Now, we briefly recall the definition of Fourier transform, see for example [16]. If $\phi \in L^{1}(X, \mathrm{~d} x ; \mathcal{Y})$, its Fourier transform $\mathcal{F}(\phi): \hat{X} \rightarrow \mathcal{Y}$ is given by

$$
\mathcal{F}(\phi)(\chi)=\int_{X} \overline{\chi(x)} \phi(x) \mathrm{d} x .
$$

We denote by $\mathrm{d} \chi$ the Haar measure on $\hat{X}$ normalized so that $\mathcal{F}$ extends to a unitary operator from $L^{2}(X, \mathrm{~d} x ; \mathcal{Y})$ onto $L^{2}(\hat{X}, \mathrm{~d} \chi ; \mathcal{Y})$. If $\mu$ is a positive measure on $X$ and $\varphi \in L^{1}(X, \mu ; \mathcal{Y})$, let $\mathcal{F}(\varphi \mu): \hat{X} \rightarrow \mathcal{Y}$ be given by

$$
\mathcal{F}(\varphi \mu)(\chi)=\int_{X} \overline{\chi(x)} \varphi(x) \mathrm{d} \mu(x) .
$$

If $\mu$ is a complex measuret on $X$, we denote $\mathcal{F}(\mu)=\mathcal{F}(h|\mu|)$ where $|\mu|$ is the total variation of $\mu$ and $h \in L^{1}(X,|\mu|)$ is the density of $\mu$ with respect to $|\mu|$.

By general properties of Fourier transform, $\mathcal{F}(\phi)$ and $\mathcal{F}(\mu)$ are bounded continuous functions on $\hat{X}$ (actually, $\mathcal{F}(\phi) \in \mathcal{C}_{0}(X ; \mathcal{Y})$ ). Moreover, $\mathcal{F}(\phi)=0$ [respectively, $\mathcal{F}(\mu)=0$ ] if and only if $\phi=0$ in $L^{1}(X, \mathrm{~d} x ; \mathcal{Y})$ [resp., $\mu=0$ ].

\footnotetext{
${ }^{4}$ That is, a $\sigma$-additive map $\mu: \mathcal{B}(X) \rightarrow \mathbb{C}$.
} 
We recall that a positive operator valued measure $(P O V M)$ on $\hat{X}$ with values in $\mathcal{Y}$ is a map $Q: \mathcal{B}(\hat{X}) \longrightarrow \mathcal{L}(\mathcal{Y})$ such that $Q(\hat{Z}) \geq 0$ for all $\hat{Z} \in \mathcal{B}(\hat{X})$, and

$$
\sum_{i} Q\left(\hat{Z}_{i}\right)=Q\left(\cup_{i} \hat{Z}_{i}\right)
$$

for every denumerable sequence of disjoint Borel sets $\left\{\hat{Z}_{i}\right\}_{i}$ where the sum converges in the weak operator topology. A positive operator valued measure $Q$ is a projection valued measure if $Q(\hat{Z})^{2}=1$ for all $\hat{Z} \in \mathcal{B}(\hat{X})$. If $\hat{f}: \hat{X} \rightarrow$ $\mathbb{C}$ is a bounded measurable function, $\int_{\hat{X}} \hat{f}(\chi) \mathrm{d} Q(\chi)$ is the unique bounded operator $\hat{f}(Q)$ defined by

$$
\left\langle\hat{f}(Q) y, y^{\prime}\right\rangle=\int_{\hat{X}} \hat{f}(\chi) \mathrm{d} Q_{y, y^{\prime}}(\chi) \quad y, y^{\prime} \in \mathcal{Y},
$$

where $Q_{y, y^{\prime}}$ is the complex measure on $\hat{X}$ given by $Q_{y, y^{\prime}}(\hat{Z})=\left\langle Q(\hat{Z}) y, y^{\prime}\right\rangle$ for all Borel subsets $\hat{Z}$.

Next theorem shows that there is a one to one correspondence between translation invariant Mercer kernels on $X$ and positive operator valued measures on $\hat{X}$. For scalar kernels this result is Bochner theorem [2]. For vector valued kernels, it is proved in [14, 15] under the weaker assumption that $K_{0}$ is a function of positive type, namely that

$$
\sum_{i, j=1}^{N} c_{i} \overline{c_{j}}\left\langle K_{0}\left(x_{i}-x_{j}\right) y, y\right\rangle \geq 0
$$

for all finite sequences $\left\{x_{i}\right\}_{i=1 \ldots N}$ in $X,\left\{c_{i}\right\}_{i=1 \ldots N}$ in $\mathbb{C}$ and $y \in \mathcal{Y}$. The fact that conditions (20) and (25) are equivalent for abelian groups is a consequence of [10, Lemma 3.1]. In the following, assuming (20), we give a proof simpler than the one provided in [14, 15].

Theorem 5. If $Q: \mathcal{B}(\hat{X}) \longrightarrow \mathcal{L}(\mathcal{Y})$ is a positive operator valued measure, then

$$
K(x, t)=\int_{\hat{X}} \chi(t-x) \mathrm{d} Q(\chi)
$$

is a translation invariant $\mathcal{Y}$-Mercer kernel on $X$. Conversely, if $K$ is a translation invariant $\mathcal{Y}$-Mercer kernel on $X$, then there exists a unique positive operator valued measure $Q$ such that (26) holds.

We say that $Q$ in (26) is the positive operator valued measure associated to the translation invariant Mercer kernel $K$. 
Proof of Theorem [5. If $Q: \mathcal{B}(\hat{X}) \longrightarrow \mathcal{L}(\mathcal{Y})$ is a positive operator valued measure, by Neumark dilation theorem [24] there exist a separable Hilbert space $\mathcal{H}$, a projection valued measure $P: \mathcal{B}(\hat{X}) \longrightarrow \mathcal{L}(\mathcal{H})$ and a bounded operator $A: \mathcal{H} \longrightarrow \mathcal{Y}$ such that

$$
Q(\hat{Z})=A P(\hat{Z}) A^{*} \quad \forall \hat{Z} \in \mathcal{B}(\hat{X}) .
$$

Let $\pi$ be the continuous unitary representation of $X$ acting on $\mathcal{H}$ given by

$$
\pi(x)=\int_{\hat{X}} \chi(x) \mathrm{d} P(\chi)
$$

see [16]. Eq. (26) then becomes $K(x, t)=A \pi_{t-x} A^{*}$, so that $K$ is a translation invariant Mercer kernel by Proposition 12 and Lemma 1.

Conversely, by Proposition 12 and Lemma 1, every translation invariant Mercer kernel is of the form $K(x, t)=A \pi_{t-x} A^{*}$ for some continuous unitary representation $\pi$ of $X$ in a separable Hilbert space $\mathcal{H}$ and some bounded operator $A: \mathcal{H} \longrightarrow \mathcal{Y}$. By SNAG theorem [16], there is then a projection valued measure $P: \mathcal{B}(\hat{X}) \longrightarrow \mathcal{L}(\mathcal{H})$ such that (28) holds and (26) follows defining the POVM $Q$ as in (27).

Finally, uniqueness of $Q$ follows from

$$
\left\langle K_{0}(x) y, y^{\prime}\right\rangle=\int_{\hat{X}} \overline{\chi(x)} \mathrm{d} Q_{y, y^{\prime}}(\chi)=\mathcal{F}\left(Q_{y, y^{\prime}}\right)(x)
$$

by injectivity of Fourier transform of measures on $\hat{X}$.

The next proposition is a useful tool to construct translation invariant Mercer kernels.

Theorem 6. Let $\hat{\nu}$ be a measure on $\hat{X}$ and $A: L^{2}(\hat{X}, \hat{\nu} ; \mathcal{Y}) \rightarrow \mathcal{Y}$ be a bounded operator. For all $y, y^{\prime} \in \mathcal{Y}$ let

$$
\left\langle K(x, t) y, y^{\prime}\right\rangle=\int_{\hat{X}} \chi(t-x)\left\langle\left(A^{*} y\right)(\chi),\left(A^{*} y^{\prime}\right)(\chi)\right\rangle \mathrm{d} \hat{\nu}(\chi) .
$$

Then $K$ is a translation invariant Mercer kernel and the corresponding reproducing kernel Hilbert space is embedded in $L^{2}(\hat{X}, \hat{\nu} ; \mathcal{Y})$ by means of the feature operator $W: L^{2}(\hat{X}, \hat{\nu} ; \mathcal{Y}) \rightarrow \mathcal{H}_{K}$

$$
\begin{aligned}
(W \hat{f})(x) & =A \hat{f}^{x} \quad \text { where } \quad \hat{f}^{x}(\chi)=\overline{\chi(x)} \hat{f}(\chi) \\
\langle(W \hat{f})(x), y\rangle & =\int_{\hat{X}} \overline{\chi(x)}\left\langle\hat{f}(\chi),\left(A^{*} y\right)(\chi)\right\rangle \mathrm{d} \hat{\nu}(\chi) .
\end{aligned}
$$

Conversely, any translation invariant Mercer kernel is of the above form for some positive measure $\hat{\nu}$ and bounded operator $A: L^{2}(\hat{X}, \hat{\nu} ; \mathcal{Y}) \rightarrow \mathcal{Y}$. 
Proof. If $\hat{\nu}$ is a measure on $\hat{X}$ and $A: L^{2}(\hat{X}, \hat{\nu} ; \mathcal{Y}) \rightarrow \mathcal{Y}$ is a bounded operator, then

$$
\left\langle Q(\hat{Z}) y, y^{\prime}\right\rangle=\int_{\hat{Z}}\left\langle\left(A^{*} y\right)(\chi),\left(A^{*} y^{\prime}\right)(\chi)\right\rangle \mathrm{d} \hat{\nu}(\chi) \quad \forall \hat{Z} \in \mathcal{B}(\hat{X}), y, y^{\prime} \in \mathcal{Y}
$$

defines a positive operator valued measure $Q: \mathcal{B}(\hat{X}) \longrightarrow \mathcal{L}(\mathcal{Y})$, since $Q(\hat{Z})=$ $A P(\hat{Z}) A^{*}$ where $P(\hat{Z})$ is the multiplication by the characteristic function of $\hat{Z}$. The kernel $K$ given in (29) is then the translation invariant Mercer kernel associated to $Q$ by (26). To prove (30), set

$$
\gamma_{x}: \mathcal{Y} \longrightarrow L^{2}(\hat{X}, \hat{\nu} ; \mathcal{Y}) \quad\left(\gamma_{x} y\right)(\chi)=\chi(x)\left(A^{*} y\right)(\chi),
$$

so that $K(x, t)=\gamma_{x}^{*} \gamma_{t}$ and

$$
\begin{aligned}
\left\langle\gamma_{x}^{*} \hat{f}, y\right\rangle & =\left\langle\hat{f}, \gamma_{x} y\right\rangle_{2}=\int_{\hat{X}}\left\langle\hat{f}(\chi), \chi(x)\left(A^{*} y\right)(\chi)\right\rangle \mathrm{d} \hat{\nu}(\chi) \\
& =\int_{\hat{X}} \overline{\chi(x)}\left\langle\hat{f}(\chi),\left(A^{*} y\right)(\chi)\right\rangle \mathrm{d} \hat{\nu}(\chi)=\left\langle A \hat{f}^{x}, y\right\rangle
\end{aligned}
$$

for all $\hat{f} \in L^{2}(\hat{X}, \hat{\nu} ; \mathcal{Y})$.

Conversely, assume that $K$ is a translation invariant Mercer kernel. We first consider the case that $\mathcal{Y}$ is infinite-dimensional. Propositions 11 and 12 show that $K$ is of the form $K(x, t)=A \pi_{t-x} A^{*}$ for some unitary continuous representatijon $\pi$ acting on a separable Hilbert space $\mathcal{H}$ and a bounded operator $A: \mathcal{H} \rightarrow \mathcal{Y}$.

A basic result of commutative harmonic analysis (see [16]) ensures that, for each $n \in \mathbb{N}_{*}:=\mathbb{N} \cup\{\infty\}$, there exist a complex separable Hilbert space $\mathcal{Y}_{n}$ of dimension $n$, and a measurable subset $\hat{X}_{n}$ of $\hat{X}$ endowed with a positive measure $\hat{\nu}_{n}$ such that the $\hat{X}_{n}$ are disjoint and cover $\hat{X}$. Without loss of generality, we can assume that $\hat{\nu}_{n}\left(\hat{X}_{n}\right) \leq 2^{-n}$ and $\hat{\nu}_{\infty}\left(\hat{X}_{\infty}\right) \leq 1$. Moreover there exists a unitary operator $U: \mathcal{H} \rightarrow \bigoplus_{n} L^{2}\left(\hat{X}_{n}, \hat{\nu}_{n}, \mathcal{Y}_{n}\right)$ such that

$$
\left(U \pi_{x} U^{*} \hat{f}_{n}\right)(\chi)=\chi(x) \hat{f}_{n}(\chi) \quad \hat{f}_{n} \in L^{2}\left(\hat{X}_{n}, \hat{\nu}_{n}, \mathcal{Y}_{n}\right) .
$$

For each $n \in \mathbb{N}_{*}$, let $J_{n}: \mathcal{Y}_{n} \rightarrow \mathcal{Y}$ be a fixed isometry, which always exists since $\mathcal{Y}$ is infinite dimensional, and consider the Hilbert space $L^{2}(\hat{X}, \hat{\nu} ; \mathcal{Y})$, where $\hat{\nu}=\sum_{n} \hat{\nu}_{n}$, which is a bounded measure by assumption on $\hat{\nu}_{n}$. Define the isometry $V: \mathcal{H} \rightarrow L^{2}(\hat{X}, \hat{\nu} ; \mathcal{Y})$ as

$$
(V u)(\chi)=J_{n}(U v)(\chi) \quad \chi \in \hat{X}_{n} .
$$

A simple calculation shows that

$$
\pi_{x}=V^{*} \hat{\lambda}_{x} V
$$


where $\left(\hat{\lambda}_{x} \hat{f}\right)(\chi)=\chi(x) \hat{f}(\chi)$ is the diagonal representation on $L^{2}(\hat{X}, \hat{\nu} ; \mathcal{Y})$. Now

$$
K(x, t)=A \pi_{t-x} A^{*}=A V^{*} \hat{\lambda}_{t-x} V A^{*} .
$$

Redefining $A=A V^{*},(29)$ is a consequence of the explicit form of $\hat{\lambda}_{x}$.

If $\mathcal{Y}$ is finite dimensional, let $(\hat{\nu}, B)$ be the pair associated to $K$ as in Proposition 13 below. Eq. (29) follows defining $A: L^{2}(\hat{X}, \hat{\nu}, \mathcal{Y}) \rightarrow \mathcal{Y}$

$$
\langle A \hat{f}, y\rangle=\int_{\hat{X}}\left\langle B(\chi)^{\frac{1}{2}} \hat{f}(\chi), y\right\rangle \mathrm{d} \hat{\nu}(\chi) .
$$

If $\mathcal{Y}=\mathbb{C}^{m}, K(x, t)$ can be regarded as a $m \times m$-matrix and $A$ is uniquely defined by a family of functions $\hat{f}_{1}, \ldots, \hat{f}_{m} \in L^{2}(\hat{X}, \hat{\nu} ; \mathcal{Y})$ through $A^{*} e_{i}=\hat{f}_{i}$. Hence, (29) becomes

$$
K(t-x)_{i j}=\int_{\hat{X}} \chi(t-x)\left\langle f_{j}(\chi), f_{i}(\chi)\right\rangle \mathrm{d} \hat{\nu}(\chi) \quad i, j=1, \ldots, m .
$$

As an application, we give the following example that generalizes the one given in [5].

Example 10. Let $X=\mathbb{R}^{d}$, regarded as vector abelian group, and $\mathcal{Y}=\mathbb{C}^{m}$. The dual group is isomorphic to $\mathbb{R}^{d}$ by means of $\chi_{p}(x)=e^{i 2 \pi x \cdot p}$. Let $\hat{\nu}=\mathrm{d} p$ be the Lebesgue measure on $\mathbb{R}^{d}$ and

$$
\hat{f}_{i}(p)=\frac{1}{(2 \pi)^{d / 4}} e^{-\sigma_{i}^{2} \frac{|p|^{2}}{2}} v_{i} \quad v_{i} \in \mathcal{Y}, \sigma_{i}>0
$$

then the translation invariant Mercer kernel given by (31) is

$$
\begin{aligned}
K(t-x)_{i j} & =\int_{\mathbb{R}^{d}} e^{i 2 \pi(t-x) \cdot p}\left\langle f_{j}(p), f_{i}(p)\right\rangle \mathrm{d} p \\
& =\frac{1}{\left(\sigma_{i}^{2}+\sigma_{j}^{2}\right)^{d / 2}} e^{-2 \pi^{2} \frac{|x-t|^{2}}{\sigma_{i}^{2}+\sigma_{j}^{2}}}\left\langle v_{j}, v_{i}\right\rangle .
\end{aligned}
$$

The example in [5] corresponds to the choice $v_{i}=v_{j}$ and $\sigma_{i}=\sigma_{j}$ for any $i, j=1, \ldots, m$.

Theorems 5 and 6 give two different characterizations of a translation invariant kernel $K$, but the POVM $Q$ defining $K$ through (26) is always unique, whereas there are many pairs $(\hat{\nu}, A)$ defining the same $K$ by (29)). These two descriptions are related observing that, given a pair $(\hat{\nu}, A)$, the scalar bounded measure $Q_{y, y^{\prime}}$ has density $\left\langle\left(A^{*} y\right)(\chi),\left(A^{*} y^{\prime}\right)(\chi)\right\rangle$ with respect 
to $\hat{\nu}$ for any $y, y^{\prime} \in \mathcal{Y}$. On the other hand, given the POVM $Q$, let $\hat{\nu}_{Q}$ be the bounded positive measure defined by

$$
\hat{\nu}_{Q}(\hat{Z})=\sum_{n} 2^{-n}\left\|y_{n}\right\|^{-2 n}\left\langle Q(\hat{Z}) y_{n}, y_{n}\right\rangle \quad \forall \hat{Z} \in \mathcal{B}(\hat{X})
$$

where $\left\{y_{n}\right\}_{n \in \mathbb{N}}$ is a dense sequence in $\mathcal{Y}$. Clearly, given $\hat{Z} \in \mathcal{B}(\hat{X}), \hat{\nu}_{Q}(\hat{Z})=0$ if and only if $Q(\hat{Z})=0$, and $\hat{\nu}_{Q}$ is uniquely defined by $Q$ up to an equivalence. Moreover, by Neumark dilation theorem, see (27), there exists an operator $A_{Q}: L^{2}\left(\hat{X}, \hat{\nu}_{Q} ; \mathcal{Y}\right) \rightarrow \mathcal{Y}$ such that the pair $\left(\hat{\nu}_{Q}, A_{Q}\right)$ gives the kernel $K$ associated with $Q$.

We notice that in general it is not true that the POVM $Q$ has an operator valued density. We recall that $Q$ has operator density if there exists a map $B: \hat{X} \longrightarrow \mathcal{L}(\mathcal{Y})$ and a positive measure $\hat{\nu}$ such that $\left\langle B(\cdot) y, y^{\prime}\right\rangle \in L^{1}(\hat{X}, \hat{\nu})$ for all $y, y^{\prime} \in \mathcal{Y}$ and

$$
\int_{\hat{Z}}\left\langle B(\chi) y, y^{\prime}\right\rangle \mathrm{d} \hat{\nu}(\chi)=Q_{y, y^{\prime}}(\hat{Z}) \quad \forall \hat{Z} \in \mathcal{B}(\hat{X}) .
$$

The following proposition will characterize the kernels having a POVM with an operator density. To prove the result, we need the following technical lemma.

Lemma 2. Let $\hat{\nu}$ be a positive measure on $\hat{X}$ and $B: \hat{X} \longrightarrow \mathcal{L}(\mathcal{Y})$ such that $\left\langle B(\cdot) y, y^{\prime}\right\rangle \in L^{1}(\hat{X}, \hat{\nu})$ for all $y, y^{\prime} \in \mathcal{Y}$. Then, the sesquilinear form

$$
\mathcal{Y} \times \mathcal{Y} \rightarrow L^{1}(\hat{X}, \hat{\nu}), \quad\left(y, y^{\prime}\right) \mapsto\left\langle B(\cdot) y, y^{\prime}\right\rangle
$$

is continuous.

Proof. For fixed $y \in \mathcal{Y}$ [resp. $y^{\prime} \in \mathcal{Y}$ ] the map $y^{\prime} \mapsto\left\langle B(\cdot) y, y^{\prime}\right\rangle$ [resp. $y \mapsto$ $\left.\left\langle B(\cdot) y, y^{\prime}\right\rangle\right]$ is continuous from $\mathcal{Y}$ into $L^{1}(\hat{X}, \hat{\nu})$ by the closed graph theorem, i.e. the application defined in (34) is separately continuous in $y$ and $y^{\prime}$. So, the closed graph theorem again assures the joint continuity.

Proposition 13. Let $\hat{\nu}$ be a positive measure on $\hat{X}$ and $B: \hat{X} \longrightarrow \mathcal{L}(\mathcal{Y})$ such that $\left\langle B(\cdot) y, y^{\prime}\right\rangle \in L^{1}(\hat{X}, \hat{\nu})$ for all $y, y^{\prime} \in \mathcal{Y}$ and $B(\chi) \geq 0$ for $\hat{\nu}$-almost all $\chi$. Then

$$
K(x, t)=\int_{\hat{X}} \chi(t-x) B(\chi) \mathrm{d} \hat{\nu}(\chi),
$$

is a translation invariant Mercer kernel, and the space $\mathcal{H}_{K}$ is embedded in $L^{2}(\hat{X}, \hat{\nu} ; \mathcal{Y})$ by means of the feature operator

$$
(W \hat{f})(x)=\int_{\hat{X}} \overline{\chi(x)} B(\chi)^{\frac{1}{2}} \hat{f}(\chi) \mathrm{d} \hat{\nu}(\chi),
$$


where both the above integrals converge in the weak sense.

If $\mathcal{Y}$ is finite dimensional or $X$ is compact, any translation invariant kernel is of the above form for some pair $(\hat{\nu}, B)$.

If $\mathcal{Y}=\mathbb{C}$, one can always assume that $B=1$ and $\hat{\nu}$ is a bounded positive measure.

Proof. Let $\hat{\nu}$ and $B$ as in the assumptions. Given a Borel subset $\hat{Z}$ of $\hat{X}$ define $Q(\hat{Z})$ as the unique bounded operator satisfying

$$
\left\langle Q(\hat{Z}) y, y^{\prime}\right\rangle=\int_{\hat{Z}}\left\langle B(\chi) y, y^{\prime}\right\rangle \mathrm{d} \hat{\nu}(\chi)
$$

The fact that $Q(\hat{Z})$ is a bounded operator follows from Lemma 2 and from the continuity of the map $L^{1}(\hat{X}, \hat{\nu}) \ni \phi \mapsto \int_{\hat{Z}} \phi(\chi) \mathrm{d} \hat{\nu}(\chi) \in \mathbb{C}$. Clearly, $Q(\hat{Z})$ is a positive operator and monotone convergence theorem implies that $\hat{Z} \mapsto Q(\hat{Z})$ is a POVM on $\hat{X}$. By construction $K(x, t)=\int_{\hat{X}} \chi(t-x) \mathrm{d} Q(\chi)$, so $K$ is a translation invariant Mercer kernel by Theorem 5. Setting

$$
\gamma_{x}: \mathcal{Y} \longrightarrow L^{2}(\hat{X}, \hat{\nu} ; \mathcal{Y}) \quad\left(\gamma_{x} y\right)(\chi)=\chi(x) B(\chi)^{1 / 2} y
$$

we see that $K(x, t)=\gamma_{x}^{*} \gamma_{t}$ and

$$
\begin{aligned}
\left\langle\gamma_{x}^{*} \hat{f}, y\right\rangle & =\left\langle\hat{f}, \gamma_{x} y\right\rangle_{2}=\int_{\hat{X}}\left\langle\hat{f}(\chi), \chi(x) B(\chi)^{1 / 2} y\right\rangle \mathrm{d} \hat{\nu}(\chi) \\
& =\int_{\hat{X}} \overline{\chi(x)}\left\langle B(\chi)^{1 / 2} \hat{f}(\chi), y\right\rangle \mathrm{d} \hat{\nu}(\chi)
\end{aligned}
$$

for all $\hat{f} \in L^{2}(\hat{X}, \hat{\nu} ; \mathcal{Y})$, from which (36) follows.

Assume now that $\mathcal{Y}$ is finite dimensional or $X$ is compact and $K$ is a translation invariant Mercer kernel. Theorem 5 ensures that there exists a POVM $Q$ on $\hat{X}$ taking value in $\mathcal{Y}$ such that $K(x, t)=\int_{\hat{X}} \chi(t-x) \mathrm{d} Q(\chi)$. If $X$ is compact, $\hat{X}$ is discrete. Let $\hat{\nu}$ be the counting measure and $B(\chi)=Q(\{\chi\})$ for all $\chi \in \hat{X}$, then $(\hat{\nu}, B)$ satisfies the required properties.

If $\mathcal{Y}$ is finite dimensional, choose $\hat{\nu}_{Q}$ as in (32). It follows that for any $y, y^{\prime} \in \mathcal{Y}$, the complex measure $Q_{y, y^{\prime}}$ has density $b_{y, y^{\prime}} \in L^{1}\left(\hat{X}, \hat{\nu}_{Q}\right)$ with respect to $\hat{\nu}_{Q}$. In particular, $b_{y, y}(\chi) \geq 0$ for $\hat{\nu}_{Q}$-almost all $\chi \in \hat{X}$. Let $y_{1}, \ldots, y_{N}$ be a basis of $\mathcal{Y}$ and by linearity extend $b_{y_{i}, y_{j}} \in L^{1}\left(\hat{X}, \hat{\nu}_{Q}\right)$ to a map $B: \hat{X} \rightarrow \mathcal{L}(\mathcal{Y})$, which clearly satisfies the required properties.

If $\mathcal{Y}=\mathbb{C}$, the claim is clear.

If $\mathcal{Y}=\mathbb{C}$, Proposition 13 is already given in [22].

We end by showing a sufficient condition ensuring that a translation invariant Mercer kernel is of the form given in Proposition 13 . 
Proposition 14. Let $K$ be a translation invariant Mercer kernel. Suppose that $\left\langle K_{0}(\cdot) y, y^{\prime}\right\rangle \in L^{1}(X, \mathrm{~d} x)$ for all $y, y^{\prime} \in \mathcal{Y}$. Let

$$
\left\langle B(\chi) y, y^{\prime}\right\rangle:=\int_{X} \chi(x)\left\langle K_{0}(x) y, y^{\prime}\right\rangle \mathrm{d} x \quad \forall y, y^{\prime} \in \mathcal{Y} .
$$

Then

(i) $B(\chi)$ is a bounded nonnegative operator for all $\chi \in \hat{X}$;

(ii) $\left\langle B(\cdot) y, y^{\prime}\right\rangle \in L^{1}(X, \mathrm{~d} x)$ for all $y, y^{\prime} \in \mathcal{Y}$;

(iii) for all $x, t \in X$,

$$
K(x, t)=\int_{\hat{X}} \chi(t-x) B(\chi) \mathrm{d} \chi
$$

where the integral converges in the weak sense.

Proof. The operator $B(\chi)$ defined in (37) is bounded as a consequence of Lemma 2 (applied to $K_{0}$ ) and of the continuity of the map $L^{1}(X, \mathrm{~d} x) \ni \phi \mapsto$ $\mathcal{F}(\phi)(\chi) \in \mathbb{C}$.

Since $\left\langle K_{0}(\cdot) y, y\right\rangle$ is a function of positive type, by Fourier inversion theorem $\langle B(\cdot) y, y\rangle \in L^{1}(\hat{X}, \mathrm{~d} \chi)$, and

$$
\left\langle K_{0}(x) y, y\right\rangle=\int_{\hat{X}} \overline{\chi(x)}\langle B(\chi) y, y\rangle \mathrm{d} \chi,
$$

which is (38).

\subsection{Universality}

In this section we study the universality problem for translation invariant kernels on an abelian group in terms of the characterization given by Theorem 5 and Proposition 13. The assumptions and notations are as in Section 5.1. To state the following result, we recall that the support of a POVM $Q$ is the complement of the largest open subset $U$ such that $Q(U)=0$.

Proposition 15. Let $K$ be a translation invariant Mercer kernel, and $Q$ its associated positive operator valued measure. If the $R K H S \mathcal{H}_{K}$ is dense in $L^{2}(X, \mu ; \mathcal{Y})$ for any probability measure $\mu$, then $\operatorname{supp}(Q)=\hat{X}$.

Proof. Suppose there is an open set $U \subset \hat{X}$ such that $Q(U)=0$. Let $\chi_{0} \in U$, so that $\chi_{0} U^{-1}$ is a neighborhood of the identity element of $\hat{X}$. Let 
$\mu$ be a probability measure 5 on $X$ such that $\operatorname{supp} \mathcal{F}(\mu) \subset \chi_{0} U^{-1}$ and set $\varphi(x)=\overline{\chi_{0}(x)} y$ with $y \in \mathcal{Y} \backslash\{0\}$. Then (26) gives

$$
\begin{aligned}
\left\langle L_{\mu} \varphi, \varphi\right\rangle & =\int_{X} \int_{X} \int_{\hat{X}} \chi(t-x) \chi_{0}(x) \overline{\chi_{0}(t)} \mathrm{d} Q_{y, y}(\chi) \mathrm{d} \mu(x) \mathrm{d} \mu(t) \\
& =\int_{\hat{X}}\left|\mathcal{F}(\mu)\left(\chi_{0} \chi^{-1}\right)\right|^{2} \mathrm{~d} Q_{y, y}(\chi)=0 .
\end{aligned}
$$

This shows that $L_{\mu}$ is not injective, i.e. $K$ is not universal.

We now characterize the universality of the kernels defined in terms of the pair $(\hat{\nu}, B)$ by means of (35) $)$.

Proposition 16. Given a positive measure $\hat{\nu}$ on $\hat{X}$ and $B: \hat{X} \longrightarrow \mathcal{L}(\mathcal{Y})$ such that $\left\langle B(\cdot) y, y^{\prime}\right\rangle \in L^{1}(\hat{X}, \hat{\nu})$ for all $y, y^{\prime} \in \mathcal{Y}$ and $B(\chi) \geq 0$ for $\hat{\nu}$-almost all $\chi$, let $K$ be the translation invariant Mercer kernel given by (35).

(i) If $\mathcal{H}_{K}$ is dense in $L^{2}(X, \mu ; \mathcal{Y})$ for any probability measure $\mu$, then both $\operatorname{supp} \hat{\nu}=\hat{X}$ and $\operatorname{supp} B=\hat{X}$.

(ii) If $\operatorname{supp} \hat{\nu}=\hat{X}$ and $B(\chi)$ is injective for $\hat{\nu}$-almost all $\chi \in \hat{X}$, then $\mathcal{H}_{K}$ is dense in $L^{2}(X, \mu ; \mathcal{Y})$ for any probability measure $\mu$.

In the case $X$ is compact also the converse holds true.

(iii) If $\mathcal{Y}=\mathbb{C}$ and $B=1, \mathcal{H}_{K}$ is dense in $L^{2}(X, \mu ; \mathcal{Y})$ for any probability measure $\mu$ if and only if $\operatorname{supp} \hat{\nu}=\hat{X}$.

Proof. Item (i) follows from Proposition 15 and (33).

Let now $\mu$ be a probability measure on $X$. Using (35), we have

$$
\begin{aligned}
\left\langle L_{\mu} \varphi, \varphi\right\rangle & =\iiint \chi(t-x)\langle B(\chi) \varphi(t), \varphi(x)\rangle \mathrm{d} \hat{\nu}(\chi) \mathrm{d} \mu(x) \mathrm{d} \mu(t) \\
& =\int_{\hat{X}}\left\langle B(\chi) \mathcal{F}(\varphi \mu)\left(\chi^{-1}\right), \mathcal{F}(\varphi \mu)\left(\chi^{-1}\right)\right\rangle \mathrm{d} \hat{\nu}(\chi) .
\end{aligned}
$$

(ii) If $B(\chi)$ is injective for almost all $\chi \in \hat{X}$ and supp $\hat{\nu}=\hat{X}$, then, by the above equation, positivity of $B(\chi)$ and the injectivity of Fourier transform, $L_{\mu} \varphi \neq 0$ if $\varphi \neq 0$ in $L^{2}(X, \mu ; \mathcal{Y})$. Therefore, $\mathcal{H}_{K}$ is dense in $L^{2}(X, \mu ; \mathcal{Y})$ for any probability measure $\mu$.

Suppose $X$ is compact, so that $\hat{X}$ is discrete. If $\mathcal{H}_{K}$ is dense in

\footnotetext{
${ }^{5}$ For example, if $V$ is a compact symmetric neighborood of the identity of $\hat{X}$ such that $V^{2} \subset \chi_{0} U^{-1}$, let $h=1_{V} * 1_{V}$, so that (up to a constant) the measure $\mathrm{d} \mu(x)=$ $\mathcal{F}^{-1}(h)(x) \mathrm{d} x=\left|\mathcal{F}^{-1}\left(1_{V}\right)(x)\right|^{2} \mathrm{~d} x$ has the required property.
} 
$L^{2}(X, \mu ; \mathcal{Y})$ for any probability measure $\mu, \operatorname{supp} \hat{\nu}=\hat{X}$ by item (i). If $\chi_{0} \in \hat{X}$ and $y \in \operatorname{ker} B\left(\chi_{0}\right)$, choose $\mathrm{d} \mu(x)=\mathrm{d} x$ and $\varphi(x)=\overline{\chi_{0}(x)} y$, so that $\mathcal{F}(\varphi \mu)(\chi)=\delta_{\chi, \chi_{0}^{-1}} y$. We thus have

$$
\left\langle L_{\mu} \varphi, \varphi\right\rangle=\left\langle B\left(\chi_{0}\right) y, y\right\rangle \hat{\nu}\left(\chi_{0}\right)=0 .
$$

Since $L_{\mu}$ is injective, this implies $\varphi=0$, i.e. $y=0$.

(iii) Since $B=1$, the 'if' part is clear from item (ii). The converse follows by item (i).

By inspecting the proofs of Propositions 15 and 16, one can easily replace $L^{2}(X, \mu ; \mathcal{Y})$ with any $L^{p}(X, \mu ; \mathcal{Y}), 1 \leq p<\infty$, in the statements. The same holds for Corollary 6 below.

Remark 3. If the translation invariant kernel $K$ is $\mathcal{C}_{0}$, then Propositions 15 and 16 characterize universality of $K$.

Remark 4. If $X=\mathbb{R}^{d}, \mathcal{Y}=\mathbb{C}$, and supp $\hat{\nu}$ is a subset of $\hat{X}=\mathbb{R}^{d}$ such that every entire function on $\mathbb{C}^{d}$ vanishing on it is identically zero, then $K$ is $\kappa$-universal (see [22, Proposition 14]). This follows by (39), taking into account that, for compactly supported $\mu$, the Fourier transform of $\varphi \mu$ can be extended to an entire function defined on $\mathbb{C}^{d}$.

In particular, if $d=1$ a sufficient condition for $\kappa$-universality is that supp $\hat{\nu}$ has an accumulation point.

Based on the above remark, we give another example of compact-universal kernel, which is not universal, see also Example 8.

Example 11. Let $K: \mathbb{R} \times \mathbb{R} \rightarrow \mathbb{C}$ be the $\mathcal{C}_{0}$-kernel

$$
K(x, t)=\int_{-1}^{1} e^{2 \pi i(t-x) p} \mathrm{~d} p=\frac{\sin 2 \pi(t-x)}{\pi(t-x)},
$$

with $\hat{\nu}$ the restriction of the Lebesgue measure to $[-1,1]$. Since the support of $\hat{\nu}$ admits an accumulation point, $K$ is compact-universal by the last remark. On the other hand since supp $\hat{\nu}$ is not the whole $\mathbb{R}, K$ is not universal by Proposition 16.

We now exhibit a particular case in which Proposition 16 applies.

Corollary 6. Let $K$ be a translation invariant Mercer kernel such that $\left\langle K_{0}(\cdot) y, y^{\prime}\right\rangle \in L^{1}(X, \mathrm{~d} x)$ for all $y, y^{\prime} \in \mathcal{Y}$. Let $B: \hat{X} \longrightarrow \mathcal{L}(\mathcal{Y})$ be as in (37). If $B(\chi)$ is injective for $\mathrm{d} \chi$-almost all $\chi$, then the reproducing kernel Hilbert space $\mathcal{H}_{K}$ is dense in $L^{2}(X, \mu ; \mathcal{Y})$ for any probability measure $\mu$.

Proof. Since the support of the Haar measure $\mathrm{d} \chi$ is $\hat{X}$, the claim is then a consequence of Proposition [16. 


\section{$6 \quad$ Examples of universal kernels}

In this section we present various examples of universal kernels, some of them has been already introduced in Section 3 .

We start with the gaussian kernel, which is a well known example of universal kernel. The first proof about universality is given [30] with a different technique and in [22] by means of the Fourier transform. In both paper only compact-universality is taken into account.

Example 12. Let $X$ be a closed subset of $\mathbb{R}^{d}, \mathcal{Y}=\mathbb{C}$ and

$$
\kappa(x, t)=e^{-\frac{\|x-t\|^{2}}{2 \sigma^{2}}} \quad x, t \in X
$$

where $\sigma>0$. Then $K$ is a $\mathcal{C}_{0}$-universal kernel.

Proof. Assume first that $X=\mathbb{R}^{d}$, regarded as abelian group, then $\kappa$ is translation invariant kernel with $\kappa_{0}$ in $\mathcal{C}_{0}\left(\mathbb{R}^{d}\right) \cap L^{1}\left(\mathbb{R}^{d}, d x\right)$. According to (37)

$$
B(p)=\sqrt{\left(2 \pi \sigma^{2}\right)^{d}} e^{-2 \pi^{2} \sigma^{2}\|p\|^{2}}
$$

where the dual group is identified with $\mathbb{R}^{d}$ by means of $\chi_{p}(x)=e^{i 2 \pi p \cdot x}$. Since $B(p)>0$ for all $p \in \mathbb{R}^{d}$, universality is a consequence of Corollary 6 . If $X$ is an arbitrary closed subset of $\mathbb{R}^{d}$ it is enough to apply Corollary 3 .

Next example is well known in functional analysis (see, for example, [3]).

Example 13. Let $X=\mathbb{R}, \mathcal{Y}=\mathbb{C}$ and let

$$
\kappa(x, t)=e^{-\pi|x-t|} .
$$

Then the kernel $\kappa$ is a $\mathcal{C}_{0}$-universal kernel and $\mathcal{H}_{\kappa}=W^{1}(\mathbb{R})$, the Sobolev space of measurable complex functions $f$ on $\mathbb{R}$ with finite norm

$$
\|f\|_{W^{1}}^{2}=\int_{X}\left[|f(x)|^{2}+\left|f^{\prime}(x)\right|^{2}\right] \mathrm{d} x
$$

where $f^{\prime}$ is the weak derivative.

Proof. The same reasoning as above, observing that $B(p)=\frac{2}{\pi+4 \pi p^{2}}>0$ for all $p \in \mathbb{R}$.

Next example characterizes universal kernels of the form $K=\kappa B$ - see Example 5. 
Example 14. Let $\kappa$ be a $\mathcal{C}_{0}$-scalar reproducing kernel and $B$ a positive operator. The kernel $K=\kappa B$ is universal if and only if $\kappa$ is universal and $B$ is injective.

Proof. We have to show that, given a probability measure $\mu, \mathcal{H}_{\kappa B}$ is dense in $L^{2}(X, \mu ; \mathcal{Y})$. The space $\mathcal{H}_{\kappa B}$ is unitarily equivalent to $\mathcal{H}_{\kappa} \otimes \operatorname{ker} B^{\perp}$ by means of $W(\varphi \otimes y)(x)=\varphi(x) B^{\frac{1}{2}} y$, see Example 5. Hence, it is enough to prove that $\mathcal{H}_{\kappa} \otimes B^{\frac{1}{2}} \mathcal{Y}$ is dense in $L^{2}(X, \mu) \otimes \mathcal{Y}$. This is the case if and only if $\mathcal{H}_{\kappa}$ is dense in $L^{2}(X, \mu)$ and $B^{\frac{1}{2}}$ has dense range, and this last condition is equivalent to the fact that $B$ is injective since $B$ is a positive operator.

The same result holds replacing $\mathcal{C}_{0}$-kernel with Mercer kernel and universality with compact-universality.

Example 15. Let $\kappa: X \times X \rightarrow \mathbb{C}$ and $\kappa^{\prime}: X^{\prime} \times X^{\prime} \rightarrow \mathbb{C}$ be two scalar $\mathcal{C}_{0}$ reproducing kernels on $X$ and $X^{\prime}$, respectively. Let $I^{\prime}$ be the identity operator on $\mathcal{H}_{\kappa^{\prime}}$.

(i) The $\mathcal{H}_{\kappa^{\prime}}$-kernel $K=\kappa I^{\prime}$ if universal if and only if $\kappa$ is universal.

(ii) Fixed a probability measure $\mu^{\prime}$ on $X^{\prime}$, the $L^{2}\left(X^{\prime}, \mu^{\prime}\right)$-kernel $\widehat{K}=\kappa L_{\mu^{\prime}}$ is universal if and only if $\kappa$ is universal and $\mathcal{H}_{\kappa^{\prime}}$ is dense in $L^{2}\left(X^{\prime}, \mu^{\prime}\right)$.

(iii) The scalar kernel $\kappa \times \kappa^{\prime}$ is universal if both $\kappa$ and $\kappa^{\prime}$ are universal.

Proof. Items (i) and (ii) follow immediately from Example 14 and Proposition 10. Item (iii) is a consequence of Proposition 9 and the density of $\mathcal{C}_{0}(X) \otimes \mathcal{C}_{0}\left(X^{\prime}\right)$ in $\mathcal{C}_{0}\left(X \times X^{\prime}\right)$.

The following class of examples is considered in [5].

Example 16. Let $X$ be a locally compact second countable abelian group. Let $\left\{B^{i}\right\}_{i=1}^{N}$ be a finite set of positive operators on $\mathcal{Y}$ and $\left\{\kappa_{0}^{i}\right\}_{i=1}^{N}$ be a finite set of scalar functions of positive type in $\mathcal{C}_{0}(X) \cap L^{1}(X, \mathrm{~d} x)$. The translation invariant kernel $K$

$$
K(x, t)=\sum_{i=1}^{N} \kappa_{0}^{i}(x-t) B^{i}
$$

is universal provided that $\cap_{i} \operatorname{ker} B^{i}=\{0\}$ and, for each $i=1, \ldots N$, there is an open dense subset $\hat{Z}^{i} \subset \hat{X}$ such that $\mathcal{F}\left(\kappa_{0}^{i}\right)>0$ on $\hat{Z}^{i}$. 
Proof. Clearly, $\left\langle K_{0}(\cdot) y, y^{\prime}\right\rangle$ is in $L^{1}(X, \mathrm{~d} x)$. Moreover, according to (37), for all $y \in \mathcal{Y}$ and $\chi \in \hat{X}$

$$
B(\chi) y=\sum_{i=1}^{N} \mathcal{F}\left(\kappa_{0}^{i}\right)\left(\chi^{-1}\right) B^{i} y
$$

Each $\widehat{Z}^{i}$ is open and dense, hence $\widehat{Z}=\cap \widehat{Z}^{i}$ is dense in $\hat{X}$. Let $\chi \in \hat{Z}$ and $y \in \mathcal{Y}$ such that $B(\chi) y=0$; then $B^{i} y=0$ for all $i=1, \ldots, N$, since every $B^{i}$ is a positive operator and $\mathcal{F}\left(\kappa_{0}^{i}\right)>0$ on $\hat{Z}^{i}$, so that by assumption $y=0$. Therefore, $K$ is universal by Corollary 6 ,

\section{A Vector valued measures}

In this appendix we describe the dual of $\mathcal{C}_{0}(X ; \mathcal{Y})$. For $\mathcal{Y}=\mathbb{C}$, it is a well known result that $\mathcal{C}_{0}(X)^{*}$ can be identified with the Banach space of complex measures on $X$. For arbitrary $\mathcal{Y}$, a similar result holds by considering the space of vector measures. If $X$ is compact, this result is due to [28] and we slightly extend it to $X$ being only locally compact. The proof we give is simpler than the original one also for $X$ compact.

Moreover, by using a version of Radon-Nikodym theorem for vector valued measures, it is possible to describe the dual of $\mathcal{C}_{0}(X ; \mathcal{Y})$ in a simpler way. Indeed, the following result holds.

Theorem 7. Let $T \in \mathcal{C}_{0}(X ; \mathcal{Y})^{*}$. There exists a unique probability measure $\mu$ on $X$ and a unique function $h \in L^{\infty}(X, \mu ; \mathcal{Y})$ such that

$$
T(f)=\int_{X}\langle f(x), h(x)\rangle \mathrm{d} \mu(x) \quad f \in \mathcal{C}_{0}(X ; \mathcal{Y})
$$

with $\|h(x)\|=\|T\|$ for $\mu$-almost all $x \in X$.

Proof. It follows combining Theorems 8 and 9 below.

Observe that, given $\mu$ and $h$ as in the statement of the theorem, if we define $T$ by (40), then $T \in \mathcal{C}_{0}(X ; \mathcal{Y})$. Hence (40) completely characterizes the dual of $\mathcal{C}_{0}(X ; \mathcal{Y})$ in terms of pairs $(\mu, h)$.

To prove the theorem, we recall some basic facts from the theory of vector valued measures (see [11, 19]). If $A \in \mathcal{B}(X)$, we denote by $\Pi(A)$ the family of partitions of $A$ into finite or denumerable disjoint Borel subsets.

Definition 3. $A$ vector measure on $X$ with values in $\mathcal{Y}$ is a mapping $\mathrm{M}$ : $\mathcal{B}(X) \longrightarrow \mathcal{Y}$ such that 
(i)

$$
\sup _{\left\{A_{i}\right\} \in \Pi(X)} \sum_{i}\left\|\mathrm{M}\left(A_{i}\right)\right\|<\infty
$$

(ii) for all $A \in \mathcal{B}(X)$ and $\left\{A_{i}\right\} \in \Pi(A)$

$$
\mathrm{M}(A)=\sum_{i} \mathrm{M}\left(A_{i}\right)
$$

where the sum converges absolutely by item (i).

If $\mathrm{M}$ is a $\mathcal{Y}$-valued vector measure on $X$, for all $A \in \mathcal{B}(X)$ we define

$$
|\mathrm{M}|(A)=\sup _{\left\{A_{i}\right\} \in \Pi(A)} \sum_{i \in I}\left\|\mathrm{M}\left(A_{i}\right)\right\| .
$$

Then, $|\mathrm{M}|$ is a bounded positive measure on $X$, called the total variation of M.

The integration of a function $f \in L^{1}(X,|\mathrm{M}| ; \mathcal{Y})$ with respect to $M$ is defined as it follows. Let $\operatorname{St}(X ; \mathcal{Y})$ be the space of functions $f=\sum_{i=1}^{n} 1_{A_{i}} v_{i}$, with $A_{i}$ disjoint Borel sets and $v_{i} \in \mathcal{Y}\left(1_{A}\right.$ is the characteristic function of the set $A$ ). For such $f$ 's, define

$$
\int_{X}\langle f(x), \mathrm{dM}(x)\rangle:=\sum_{i=1}^{n}\left\langle v_{i}, \mathrm{M}\left(A_{i}\right)\right\rangle \text {. }
$$

Since

$$
\left|\sum_{i=1}^{n}\left\langle v_{i}, \mathrm{M}\left(A_{i}\right)\right\rangle\right| \leq \sum_{i}\left\|v_{i}\right\|\left\|\mathrm{M}\left(A_{i}\right)\right\| \leq \sum_{i}|\mathrm{M}|\left(A_{i}\right)\left\|v_{i}\right\|=\|f\|_{1},
$$

the integral (41) extends to a bounded functional on $L^{1}(X,|\mathrm{M}| ; \mathcal{Y})$, which is denoted again by $\int_{X}\langle f(x), \mathrm{dM}(x)\rangle$. By Theorem 4.1 in [19], then there exists $h \in L^{\infty}(X,|\mathrm{M}| ; \mathcal{Y})$ such that

$$
\int\langle f(x), \operatorname{dM}(x)\rangle=\int\langle f(x), h(x)\rangle \mathrm{d}|\mathrm{M}|(x) \quad \forall f \in L^{1}(X,|\mathrm{M}| ; \mathcal{Y}),
$$

and $\|h(x)\|=1$ for $|\mathrm{M}|$-almost all $x$. These facts are collected in the following theorem.

Theorem 8 (Radon-Nikodym). If $\mathrm{M}$ is a $\mathcal{Y}$-valued vector measure on $X$, there exists a unique $|\mathrm{M}|$-measurable function $h: X \longrightarrow \mathcal{Y}$ such that $\|h(x)\|=$ 1 for $|\mathrm{M}|$-almost all $x$ and

$$
\int_{X}\langle f(x), \operatorname{dM}(x)\rangle=\int_{X}\langle f(x), h(x)\rangle \mathrm{d}|\mathrm{M}|(x) \quad \forall f \in L^{1}(X,|\mathrm{M}| ; \mathcal{Y}) .
$$


The function $h$ is called the density of $\mathrm{M}$ with respect to $|\mathrm{M}|$.

We denote by $M(X ; \mathcal{Y})$ the space of $\mathcal{Y}$-valued vector measures on $X$. The space $M(X ; \mathcal{Y})$ is a Banach space with respect to the norm

$$
\|\mathrm{M}\|=|\mathrm{M}|(X)
$$

(see [11]). If $\mathcal{Y}=\mathbb{C}$, we let $M(X)=M(X ; \mathbb{C})$. The next duality theorem is shown in [28] for $X$ compact - see also [11].

Theorem 9. If $\mathcal{C}_{0}(X ; \mathcal{Y})$ is endowed with the Banach space topology induced by the uniform norm, then $\mathcal{C}_{0}(X ; \mathcal{Y})^{*}=M(X ; \mathcal{Y})$, the duality being given by

$$
\langle f, \mathrm{M}\rangle=\int_{X}\langle f(x), \mathrm{dM}(x)\rangle \quad \forall f \in \mathcal{C}_{0}(X ; \mathcal{Y}), \mathrm{M} \in M(X ; \mathcal{Y}) .
$$

Proof. By Theorem 8 , it is clear that, if $\mathrm{M} \in M(X ; \mathcal{Y})$, then

$$
T_{\mathrm{M}}(f)=\int_{X}\langle f(x), \mathrm{dM}(x)\rangle=\int_{X}\langle f(x), h(x)\rangle \mathrm{d}|\mathrm{M}|(x)
$$

defines a bounded functional $T_{\mathrm{M}}$ on $\mathcal{C}_{0}(X ; \mathcal{Y})$.

Clearly $\left\|T_{\mathrm{M}}\right\| \leq\|\mathrm{M}\|$. To show that $\left\|T_{\mathrm{M}}\right\|=\|\mathrm{M}\|$, fix by Lusin theorem a function $g \in \mathcal{C}_{0}(X ; \mathcal{Y})$ such that $g(x)=h(x)$ for $x \in X \backslash Z, Z$ being a $|\mathrm{M}|$-measurable set with $|\mathrm{M}|(Z)<\epsilon$, and $\|g\|_{\infty} \leq\|h\|_{|M|, \infty}=1$. For $\epsilon$ small enough, we then have

$$
|\mathrm{M}|(X)-2 \epsilon<|\mathrm{M}|(X \backslash Z)-|\mathrm{M}|(Z) \leq\left|\int_{X}\langle g(x), h(x)\rangle \mathrm{d}\right| \mathrm{M}|(x)| \leq|\mathrm{M}|(X) .
$$

This shows that $\left\|T_{\mathrm{M}}\right\|=\|\mathrm{M}\|$.

Suppose now $T \in \mathcal{C}_{0}(X ; \mathcal{Y})^{*}$. For $v \in \mathcal{Y}$, let $i_{v}: \mathcal{C}_{0}(X) \longrightarrow \mathcal{C}_{0}(X ; \mathcal{Y})$ be the bounded operator given by

$$
\left[i_{v}(\varphi)\right](x)=\varphi(x) v
$$

Since $T i_{v} \in \mathcal{C}_{0}(X)^{*}$, by Riesz theorem there exists a measure $\mu_{v} \in M(X)$ such that

$$
T i_{v}(\varphi)=\int_{X} \varphi(x) \mathrm{d} \mu_{v}(x) \quad \text { and } \quad\left\|T i_{v}\right\|=\left\|\mu_{v}\right\| .
$$

For all $A \in \mathcal{B}(X)$, let $\mathrm{M}(A)$ be the vector in $\mathcal{Y}$ such that

$$
\langle v, \mathrm{M}(A)\rangle=\mu_{v}(A)
$$


$\left(\mathrm{M}(A)\right.$ is well defined, since $\left.\left|\mu_{v}(A)\right| \leq\left\|\mu_{v}\right\|=\left\|T i_{v}\right\| \leq\|T\|\|v\|\right)$.

We now show that, if $A \in \mathcal{B}(X)$ and $\left\{A_{i}\right\} \in \Pi(A)$, then

$$
\sum_{i}\left\|\mathrm{M}\left(A_{i}\right)\right\| \leq\|T\|
$$

so that item (i) of Definition 3 holds. It is enough to prove it for all finite partitions $\left\{A_{i}\right\}_{i=1 \ldots n}$. Let $v_{i}=\mathrm{M}\left(A_{i}\right) /\left\|\mathrm{M}\left(A_{i}\right)\right\|$ (we set $v_{i}=0$ whenever $\left.\mathrm{M}\left(A_{i}\right)=0\right)$. We have

$$
\sum_{i}\left\|\mathrm{M}\left(A_{i}\right)\right\|=\sum_{i}\left\langle v_{i}, \mathrm{M}\left(A_{i}\right)\right\rangle=\sum_{i} \mu_{v_{i}}\left(A_{i}\right) .
$$

Set $\nu=\sum_{i}\left|\mu_{v_{i}}\right|$, which is $\nu$ a bounded positive measure, and every $\mu_{v_{i}}$ has density with respect to $\nu$. For all $i=1 \ldots n$, fix a sequence $\left\{\varphi_{j}^{(i)}\right\}_{j \in \mathbb{N}}$ in $\mathcal{C}_{c}(X)$ such that $\lim _{j} \varphi_{j}^{(i)}(x)=1_{A_{i}}(x)$ for $\nu$-almost all $x$. Define

$$
\psi_{j}(x)=\left[1 \vee \sum_{k=1}^{n}\left|\varphi_{j}^{(k)}(x)\right|\right]^{-1} \sum_{i=1}^{n} \varphi_{j}^{(i)}(x) v_{i}
$$

Then, $\psi_{j} \in \mathcal{C}_{c}(X ; \mathcal{Y})$, and $\left\|\psi_{j}(x)\right\| \leq 1$ for all $x$. Moreover,

$$
\left|\left[1 \vee \sum_{k=1}^{n}\left|\varphi_{j}^{(k)}(x)\right|\right]^{-1} \varphi_{j}^{(i)}(x)\right| \leq 1 \quad \forall x, i
$$

and

$$
\lim _{j}\left[1 \vee \sum_{k=1}^{n}\left|\varphi_{j}^{(k)}(x)\right|\right]^{-1} \varphi_{j}^{(i)}(x)=1_{A_{i}}(x) \text { for } \nu \text {-almost all } x .
$$

Therefore

$$
\begin{aligned}
& \left|\sum_{i}\left\|\operatorname{M}\left(A_{i}\right)\right\|-T \psi_{j}\right| \\
& =\left|\sum_{i}\left\{\mu_{v_{i}}\left(A_{i}\right)-T i_{v_{i}}\left(\left[1 \vee \sum_{k}\left|\varphi_{j}^{(k)}\right|\right]^{-1} \varphi_{j}^{(i)}\right)\right\}\right| \\
& \quad \leq \sum_{i}\left|\int_{X}\left\{1_{A_{i}}(x)-\left[1 \vee \sum_{k}\left|\varphi_{j}^{(k)}(x)\right|\right]^{-1} \varphi_{j}^{(i)}(x)\right\} \mathrm{d} \mu_{v_{i}}(x)\right| \\
& \quad \stackrel{j \rightarrow \infty}{\longrightarrow} 0
\end{aligned}
$$


by dominated convergence theorem. On the other hand, $\left|T \psi_{j}\right| \leq\|T\|\left\|\psi_{j}\right\|_{\infty} \leq$ $\|T\|$. It follows that $\sum_{i=1}^{n}\left\|\mathrm{M}\left(A_{i}\right)\right\| \leq\|T\|$, as claimed.

We now show that

$$
\mathrm{M}(A)=\sum_{i} \mathrm{M}\left(A_{i}\right)
$$

(absolutely) for all $A \in \mathcal{B}(X)$ and $\left\{A_{i}\right\} \in \Pi(A)$. We have just proved that the right hand side is absolutely convergent, and the equality follows by

$$
\left\langle v, \sum_{i} \mathrm{M}\left(A_{i}\right)\right\rangle=\sum_{i} \mu_{v}\left(A_{i}\right)=\mu_{v}(A)=\langle v, \mathrm{M}(A)\rangle \quad \forall v \in \mathcal{Y} .
$$

Therefore, $\mathrm{M}$ is a $\mathcal{Y}$-valued measure. It remains to show that $T=T_{\mathrm{M}}$. Let $h$ and $|\mathrm{M}|$ be associated to $\mathrm{M}$ as in Radon-Nikodym theorem. Then, for any Borel set $A \subset X$, we have $\mu_{v}(A)=\int_{A}\langle v, h(x)\rangle \mathrm{d}|\mathrm{M}|(x)$, from which it follows that $\mu_{v}$ has density $\langle v, h(x)\rangle$ with respect to $|\mathrm{M}|$. For $\varphi \in \mathcal{C}_{c}(X)$ and $v \in \mathcal{Y}$, we thus have

$$
T(\varphi v)=\int_{X} \varphi(x) \mathrm{d} \mu_{v}(x)=\int_{X}\langle\varphi(x) v, h(x)\rangle \mathrm{d}|\mathrm{M}|(x)=T_{\mathrm{M}}(\varphi v) .
$$

Then, $T=T_{\mathrm{M}}$ by density of $\mathcal{C}_{c}(X) \otimes \mathcal{Y}$ in $\mathcal{C}_{0}(X ; \mathcal{Y})$.

Acknowledgment. This work has been partially supported by the FIRB project RBIN04PARL and by the the EU Integrated Project Health-e-Child IST-2004-027749.

\section{References}

[1] N. Aronszajn. Theory of reproducing kernels. Trans. Amer. Math. Soc., 68:337-404, 1950.

[2] S. Bochner. Lectures on Fourier integrals. With an author's supplement on monotonic functions, Stieltjes integrals, and harmonic analysis. Translated by Morris Tenenbaum and Harry Pollard. Annals of Mathematics Studies, No. 42. Princeton University Press, Princeton, N.J., 1959 .

[3] H. Brézis. Analyse fonctionnelle : théorie et applications. Dunod, Paris, 1983.

[4] A. Caponnetto and E. De Vito. Optimal rates for the regularized leastsquares algorithm. Found. Comput. Math., 7(3):331-368, 2007. 
[5] A. Caponnetto, C. A. Micchelli, M. Pontil, and Y. Ying. Universal kernels for multi-task learning. J. Mach. Learn. Res., 2008 (accepted). Preprint available at http://eprints.pascal-network.org/archive/00003780/.

[6] C. Carmeli, E. De Vito, and A. Toigo. Vector valued reproducing kernel Hilbert spaces of integrable functions and Mercer theorem. Anal. Appl. (Singap.), 4(4):377-408, 2006.

[7] J. B. Conway. A course in functional analysis, volume 96 of Graduate Texts in Mathematics. Springer-Verlag, New York, second edition, 1990.

[8] F. Cucker and S. Smale. On the mathematical foundations of learning. Bull. Amer. Math. Soc. (N.S.), 39(1):1-49 (electronic), 2002.

[9] F. Cucker and D.-X. Zhou. Learning theory: an approximation theory viewpoint. Cambridge Monographs on Applied and Computational Mathematics. Cambridge University Press, Cambridge, 2007. With a foreword by Stephen Smale.

[10] E. B. Davies. Quantum theory of open systems. Academic Press [Harcourt Brace Jovanovich Publishers], London, 1976.

[11] J. Diestel and J. J. Uhl, Jr. Vector measures. American Mathematical Society, Providence, R.I., 1977. With a foreword by B. J. Pettis, Mathematical Surveys, No. 15.

[12] T. Evgeniou, C. A. Micchelli, and M. Pontil. Learning multiple tasks with kernel methods. J. Mach. Learn. Res., 6:615-637 (electronic), 2005.

[13] T. Evgeniou, M. Pontil, and T. Poggio. Regularization networks and support vector machines. Adv. Comput. Math., 13(1):1-50, 2000.

[14] P. L. Falb. On a theorem of Bochner. Inst. Hautes Études Sci. Publ. Math., 36:59-67, 1969.

[15] P. L. Falb and U. Haussmann. Bochner's theorem in infinite dimensions. Pacific J. Math., 43:601-618, 1972.

[16] G. B. Folland. A course in abstract harmonic analysis. Studies in Advanced Mathematics. CRC Press, Boca Raton, FL, 1995.

[17] H. Führ. Abstract harmonic analysis of continuous wavelet transforms, volume 1863 of Lecture Notes in Mathematics. Springer-Verlag, Berlin, 2005 . 
[18] L. Györfi, M. Kohler, A. Krzyżak, and H. Walk. A distribution-free theory of nonparametric regression. Springer Series in Statistics. SpringerVerlag, New York, 2002.

[19] S. Lang. Real and functional analysis, volume 142 of Graduate Texts in Mathematics. Springer-Verlag, New York, third edition, 1993.

[20] Y. Lee, Y. Lin, and G. Wahba. Multicategory support vector machines. In Proceedings of the 33rd Symposium on the Interface, 2001.

[21] C. A. Micchelli and M. Pontil. On learning vector-valued functions. Neural Comput., 17(1):177-204, 2005.

[22] C. A. Micchelli, Y. Xu, and H. Zhang. Universal kernels. J. Mach. Learn. Res., 7:2651-2667, 2006.

[23] G. Pedrick. Theory of reproducing kernels for hilbert spaces of vector valued functions. Technical report, Kansas Univ Lawrence, 1957.

[24] F. Riesz and B. Sz.-Nagy. Functional analysis. Dover Books on Advanced Mathematics. Dover Publications Inc., New York, 1990. Translated from the second French edition by Leo F. Boron, Reprint of the 1955 original.

[25] S. Saitoh. Theory of reproducing kernels and its applications, volume 189 of Pitman Research Notes in Mathematics Series. Longman Scientific \& Technical, Harlow, 1988.

[26] B. Schoelkopf and J. Smola. Learning with Kernels. The MIT Press, Cambridge, MA, 2002.

[27] L. Schwartz. Sous-espaces hilbertiens d'espaces vectoriels topologiques et noyaux associés (noyaux reproduisants). J. Analyse Math., 13:115256,1964 .

[28] I. Singer. Linear functionals on the space of continuous mappings of a compact Hausdorff space into a Banach space. Rev. Math. Pures Appl., 2:301-315, 1957.

[29] S. Smale and D.-X. Zhou. Shannon sampling. II. Connections to learning theory. Appl. Comput. Harmon. Anal., 19(3):285-302, 2005.

[30] I. Steinwart. On the influence of the kernel on the consistency of support vector machines. Journal of Machine Learning Research, 2:67-93, Nov. 2001. 
[31] H.-W. Sun and D.-X. Zhou. Reproducing kernel Hilbert spaces associated with analytic translation-invariant Mercer kernels. J. Fourier Anal. Appl., 14(1):89-101, 2008.

[32] D. X. Zhou. Density problem and approximation error in learning theory. Technical report, City University of Hong Kong, 2003. 